\title{
Management of Dry Gas Desulfurization By-Products in Underground Mines
}

\author{
Quarterly Report
}

\author{
October 1 - December 31, 1996
}

Work Performed Under Contract No.: DE-FC21-93MC30252

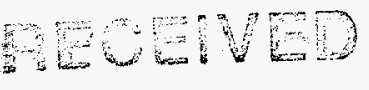

MAR 31 \%

OSTI

For

U.S. Department of Energy

Office of Fossil Energy

Morgantown Energy Technology Center

P.O. Box 880

Morgantown, West Virginia 26507-0880

By

Southern Illinois University of Carbondale

Department of Mining

Carbondale, Illinois 62901-6603 


\section{DISCLAMMER}

Portions of this document may be illegible in electronic image products. Images are produced from the best available original document. 


\section{Disclaimer}

This report was prepared as an account of work sponsored by an agency of the United States Government. Neither the United States Government nor any agency thereof, nor any of their employees, makes any warranty, express or implied, or assumes any legal liability or responsibility for the accuracy, completeness, or usefulness of any information, apparatus, product, or process disclosed, or represents that its use would not infringe privately owned rights. Reference herein to any specific commercial product, process, or service by trade name, trademark, manufacturer, or otherwise does not necessarily constitute or imply its endorsement, recommendation, or favoring by the United States Government or any agency thereof. The views and opinions of authors expressed herein do not necessarily state or reflect those of the United States Government or any agency thereof. 


\section{MANAGEMENT OF DRY FLUE GAS DESULFURIZATION BY-PRODUCTS IN UNDERGROUND MINES}

Technical Progress Report - October 1, 1996 - December 31, 1996

\section{CONTENTS}

Section 1

Introduction and Summary.

Section 2

Environmental Characterization.

Dr. Bradley Paul - Co-Principal Investigator

Section 3

Materials Handling \& System Economics

Dr. Hasan Sevim - Co-Principal Investigator

Section 4

Environmental Assessment \& Geotechnical Stability.

Dr. Steven Esling - Co-Principal Investigator

Section 5

Underground Placement.

Dr. Y.P. Chugh - Co-Principal Investigator

Eric Powell - Researcher

Gary Wangler - Researcher

Section 6

Plans For Next Quarter

Edwin M. Thomasson

Program Manager

Appendix.

Paper to be presented at the Annual Meeting of the Society of Mining Engineers by Dr. Hasan Sevim 


\section{MANAGEMENT OF DRY FLUE GAS DESULFURIZATION BY-PRODUCTS IN UNDERGROUND MINES}

Technical Progress Report - October 1, 1996 - December 31, 1996

\section{TABLES}

Section 4

Table 4.1 Screened interval and depth to water measurement in monitoring wells $2 \mathrm{~A}, 3$, and 4

Table 4.2 Screened interval and depth to water measurement in monitoring wells 5 and 6 ....

Table 4.3 Depth to water measurement, samples collected, and flow-through-cell parameters for deep wells in each well nest, February 6 to March 11, 1996.

Table 4.4 Depth to water measurement, samples collected, and flow-through-cell parameters for deep wells in each well nest, March 29 to May 15, 1996

Table 4.5 Depth to water measurement, samples collected, and flow-through-cell parameters for deep wells in each well nest, June 23 to October 12. 1996.

Table 4.6 Depth to water measurement, samples collected, and flow-through-cell parameters for deep wells in each well nest, November 12, 1996 to January 7, 1997

Table 4.7 Laboratory analysis of cation constituents, February and March sampling, 1996.

Table 4.8 Laboratory analysis of anion constituents, February and March sampling, 1996.

Table 4.9 Laboratory analysis of cation constituents, April, May, and June, 1996

Table 4.10 Laboratory analysis of anion constituents, April, May, and June, 1996. 
Table 4.11 Laboratory analysis of cation constituents, July through November sampling, 1996.

Table 4.12 Laboratory analysis of anion constituents, July through November sampling, 1996.

\section{FIGURES}

\section{Section 5}

Figure 5.1 Overall view of pneumatic placement unit under construction.... 23

Figure $5.2 \quad$ Close-up of the ribbon screw conveyors in the fly ash vessels.... 23 


\section{SECTION 1}

INTRODUCTION AND SUMMARY 


\title{
MANAGEMENT OF DRY FLUE GAS DESULFURIZATION BY-PRODUCTS IN UNDERGROUND MINES
}

\author{
Technical Progress Report - October 1, 1996 - December 31, 1996
}

\section{INTRODUCTION AND SUMMARY}

On September 30,1993 the U.S. Department of Energy - Morgantown Energy Technology Center (DOE-METC) and Southern Illinois University at Carbondale (SIUC) entered into a cooperative research agreement entitled "Management of Dry Flue Gas Desulfurization By-Products in Underground Mines" (Contract DE-FC21-93MC 30252). Under the agreement SIUC will develop and demonstrate two technologies for the placement of coal combustion by-products in abandoned underground coal mines, and will assess the environmental impact of these technologies for the management of coal combustion by-products. The two technologies for the underground placement that will be developed and demonstrated are: (1) pneumatic placement using virtually dry coal combustion by-products, and (2) hydraulic placement using a "paste" mixture of combustion by-products with about $70 \%$ solids.

Phase II of the overall program began April 1, 1996. The principal objective of Phase II is to develop and fabricate the equipment for both the pneumatic and hydraulic placement technologies, and to conduct a limited, small-scale "shakedown" test of the pneumatic and hydraulic placement equipment. The shakedown test originally was to take place on the surface, in trenches dug for the tests. However, after a thorough study it was decided, with the concurrence of DOE-METC, to drill additional injection wells and conduct the shakedown tests underground. This will allow a more thorough test of the placement equipment.

During the current quarter the main thrust was the acquisition of the various components of the placement systems, and the assembly of the systems. A mixing plant for mixing the hydraulic by-products was acquired from the highway department of Montgomery County, Illinois, and at the end of the quarter the plant had been disassembled, transported to the demonstration site at the Peabody No. 10 mine near Pawnee, Illinois, and the re-assembly and re-configuration of the plant begun. It is anticipated that the plant will be completely re-assembled by the end of January, 1997. Also, a high-capacity pump for the hydraulic placement had been located. 
The pneumatic placement equipment is being built "from scratch", and much of the quarter was devoted to the acquisition of various component parts from suppliers throughout Southern Illinois. However, by the end of the quarter actual construction of the equipment had begun at the SIUC Carterville, Illinois facility. Again, it is anticipated that the construction of the pneumatic equipment will be completed by the end of January, 1997. The completed assembly will be transported to the demonstration site by flat-bed truck.

Permits for both the "shakedown" tests for the full-scale demonstration under Phase III of the program have been received from the appropriate State of Illinois agencies. Peabody Coal Company, one of the cooperating organizations, obtained the permits; however, laboratory data developed by project personnel was used by Peabody in the permit applications.

The Christmas holidays and the closure of the University during the last week of December curtailed some activities. However, the overall program remains essentially on schedule. 


\title{
SECTION 2
}

\section{ENVIRONMENTAL CHARACTERIZATION}

\author{
DR. BRADLEY PAUL \\ CO-PRINCIPAL INVESTIGATOR
}




\title{
MANAGEMENT OF DRY FLUE GAS DESULFURIZATION BY-PRODUCTS IN UNDERGROUND MINES
}

\author{
Technical Progress Report - October 1, 1996 - December 31, 1996
}

\section{ENVIRONMENTAL CHARACTERIZATION}

Environmental characterization is a support activity designed to assure that the material placed underground as part of the demonstration is unlikely to be harmful to the environment. Also, the data developed will provide information needed to obtain the necessary permits. In previous quarters the data for much of the assessment has been developed and the data for the permits has been acquired and delivered. Permits are now in place as the underground injection equipment is readied.

Few aspects of underground injection of dry FGD by-products would raise any reasonable concerns of damage to the groundwater, however, previous work indicates relatively high levels of alkali metals and sulfate. While such elements do not threaten toxic effects, high dissolved solids and sulfate may threaten the usability of water with respect to hardness and taste.

Much of the Illinois coal basin has relatively shallow brines, and the higher dissolved solids leachate possible from dry FGD placement would be quite compatible with such waters. At the demonstration site the nearest "aquifer unit" has a permeability in the $10^{\wedge}-9 \mathrm{~cm} / \mathrm{sec}$ range with moderately briny water. This unit has been discussed in much greater detail in the report of Dr. Steve Esling who has monitored the onsite hydrogeology and water quality. Thus dissolved solids and taste is no threat to the groundwater at the demonstration site.

While disposal of FGD by-products in a manner that does not consume large amounts of surface land is an objective of the DOE study, it is intended that the type of placement envisioned provide added benefits such as subsidence control and control of acid mine drainage. Within the Illinois Basin region, subsidence is a greater concern than acid mine drainage. Many subsidence problem sites in the Illinois Basin involve relatively shallow mines producing sink-hole subsidence with disastrous results to surface structures. Conventional grouts have been used to stabilize such problems but with costs in the $\$ 60 /$ ton range, management of subsidence may be more costly than the damage it causes. Projected costs for the system being developed in this study are less than $1 / 6^{\text {th }}$ the cost of convention grouting and would place subsidence control in economically achievable range. The environmental issue raised by the potential to generalize the technology being developed in this study is that the shallow mines with some of the most pressing needs for subsidence control are also in contact with prime high quality aquifers which in many cases are providing water for human use and consumption. Degenerating these water resources with high dissolved solids and sulfates would be acceptable. 
For a material that produces high dissolved solids-high sulfate leachate to significantly degrade water quality there must be enough leachate relative to the surrounding water supply to raise the solids and sulfates content in the entire water supply. Two variables key to this potential are the amount of water available at the site, and the amount of high dissolved solids leachate that the FGD backfill produces. The amount of water available in a water supply and the hydraulic gradient available to drive water through the FGD material are all site specific considerations. The ease with which flow can be forced through a material (it's permeability) is a material characteristic. Permeability was a variable that was incidentally studied as part of early work on environmental characterization. Now permeability is the key to how many settings FGD backfilling would be suitable. Synthetic gypsum with fly ash to promote flow and setting (the hydraulic mix formula) has a modest permeability around $10^{\wedge}-4$ to $10^{\wedge}-5 \mathrm{~cm} / \mathrm{sec}$. The values are a little low for many of the more open aquifers, but are high enough to allow significant volumes of leachate to join the water supply in an area.

By contrast, the FBC ash mixes used for pneumatic backfilling may be very low in permeability. On earlier "Rapid Age" tests, when the pneumatic mix was packed into the column it was not possible to recover leachate for a sample. The original rapid age apparatus could measure permeability's in the $10^{\wedge}-6 \mathrm{~cm} / \mathrm{sec}$ range. In response to the problem, the Rapid Age apparatus was filled by loosely pouring the material into place. This procedure produced leachate, but the FBC materials is prone to swell and secondary mineral formation. The initial permeability falls and after about two weeks to two months the rapid age columns again shut themselves down. The result suggests that the pneumatic backfill will produce so little leachate that near surface water resources would not be threatened, since the potable near surface water resources in the Illinois Basin generally are in fairly permeable strata. In other work, however, it has been found that combustion by-products will frequently loose permeability in a column leaching setting and then become much more permeable later. Some studies of FBC materials suggest that these materials may not be durable and that some of the changes could cause major increases in permeability. To observe such long trends, however, some water must continue to move through the material. This requires a more powerful rapid aging apparatus that can maintain leaching even as initial permeability is lost.

In the last report the concept for the new rapid aging column was given. The column is two feet in diameter and two feet long with limited tubing and connections. The device should be able to product $25 \mathrm{ml}$ of leachate per day even with permeability's in the $10^{\wedge}-8 \mathrm{~cm} / \mathrm{sec}$ range. This quarter the details of the device have been worked out and manufacturing has begun. There will be only two columns, both of them testing the pneumatic mix material, since this would be the material of choice for high quality groundwater settings. (The old rapid age apparatus produced sufficient data on the hydraulic mix). The rapid age column is made of galvanized steel culvert pipe with a welded lip and rubber sealed bolted cap. The pipe will be painted on the inside with an inert epoxy resin, 
similar to the ceramics used to coat kitchen sinks (anti-rust paint is considered to be too porous). The apparatus will have a direct feed from the nitrogen pressure tank to limit the junctions that ultimately controlled the maximum pressure that could be applied to the earlier rapid age assembly.

As the rapid age column is built, one of the questions that must be dealt with is how to pack the column when it arrives. It is known that packing profoundly effects permeability with a Proctor compacted material having very little permeability initially, and a loose poured material having at least some initial permeability. In the mine material will be blown into place, with air pulses used to form passages and keep material moving. No information could be found on what kind of packing density such handling would produce. It was hoped to use cores from the material in the phase II surface demonstration, but the Phase II surface demonstration will be used to check the operation of the deep pneumatic placement equipment. (The problem is that plywood sealed surface trenches will not be air tight and the air pulses used to keep the flow of material open will leak out or even tear-up the model). Even if cores are produced by the final phase III of the project, the packing density will be available only at the end of the study and will not be able to provide data for long term testing on aging and permeability. To overcome this problem, data from materials placement modeling is being used. A Plexiglas mine has been constructed and outfitted with a hopper and pulsed injection system similar in function to the commercial scale device being built. Sections of the model will be removed and weighed to determine the achieved density. Of course not all aspects of the model are to scale, and there is some risk of excessive edge effects. The extent or existence of these effects can be checked at the end of the program when cores of the placed material are taken. 
SECTION 3

\title{
MATERIALS HANDLING AND SYSTEM ECONOMICS
}

\author{
DR. H. SEVIM \\ CO-PRINCIPAL INVESTIGATOR
}




\title{
MANAGEMENT OF DRY FLUE GAS DESULFURIZATION BY-PRODUCTS IN UNDERGROUND MINES
}

\author{
Technical Progress Report - October 1, 1996 - December 31, 1996
}

\section{MATERIALS HANDLING AND SYSTEM ECONOMICS}

\section{OBJECTIVES}

The objectives of the materials handling research are: 1) To identify the systems that are technically, economically, and environmentally feasible in handling and transporting the coal combustion by-products from the power plant to the injection site, 2) To demonstrate the operation of one or two of the identified systems.

The objectives of the system economics research are: 1) To conduct economic analyses of the selected materials handling and underground residue placement systems, and 2) To develop a generalized "Economic Evaluation" model that can be used in evaluating various types of materials handling and placement systems for different distances and tonnages.

The term "materials handling" in this research project includes loading, transporting, unloading, and temporary storage of the dry coal combustion by-products for the purpose of disposing them into abandoned areas of the underground coal mines in Illinois. Materials handling systems have been analyzed in four consecutive modules: 1) storage, handling and loading of the residues at the plant site, 2) transportation from the plant to the mine site, 3) unloading, handling and storage at the mine site, and 4) transportation from the mine site to the injection site.

In the past, several transportation and handling technologies were evaluated. These technologies can be classified under two categories:

1. Existing Technologies:

1.1 Pneumatic Trucks (PT)

1.2 Pressure differential rail cars (PD-car)

1.3 Open Hopper Coal Cars

1.4 Tarped Rear-Dump Trucks

1.5 Bottom-Dump Container Trucks 
2. Adaptable and Futuristic Technologies:

\subsection{Collapsible Intermodal Containers (CIC)}

2.2 Cylindrical Intermodal Tanks (CIT)

2.3 Intermodal Steel Containers

2.4 Coal Hopper Cars with Automatic Retractable Tarping

2.5 Covered Hopper Cars - Grain Cars

The first two technologies in each category were found to be environmentally friendly, and, therefore, operating policies for these technologies were developed and engineering and economic analyses were conducted.

\section{SUMMARY OF ACTIVITIES}

During the last quarter, the topical report in materials handling and systems economics was being prepared. In the report the above mentioned technologies are presented in detail; the design and economic models developed for the environmentally friendly technologies are described; the favorable operating range of each technology was determined in terms of distance and tonnage using the developed software; and a case study for Peabody \#10 Mine in Pawnee, Illinois, the future site of the underground placement of by-products, was conducted.

Also in this quarter a technical paper titled "Promoting Illinois Coal Utilization Through Underground Disposal of Combustion By-products" was prepared. The paper will be presented at the 1997 SME Annual Meeting to be held February 24-27,1997, in Denver, Colorado. A copy of the paper is given in the Appendix of this report.

\section{PLANS FOR THE NEXT QUARTER}

In the next quarter two major tasks will be undertaken:

1. Finalize the topical report.

2. Complete the user's manual for the developed software on engineering design and economic evaluation of the selected coal combustion by-product transportation, handling, and underground placement technologies. 


\title{
SECTION 4
}

\author{
ENVIRONMENTAL ASSESSMENT \\ \& GEOTECHNICAL STABILITY \\ Dr. S. Esling \\ CO-PRINCIPAL INVESTIGATOR
}


MANAGEMENT OF DRY FLUE GAS DESULFURIZATION

BY-PRODUCTS IN UNDERGROUND MINES

Technical Progress Report - October 1, 1996 - December 31, 1996

\section{ENVIRONMENTAL ASSESSMENT AND GEOTECHNICAL STABILITY AND SUBSIDENCE IMPACTS}

\section{Accomplishments}

Researchers from SIUC and the ISGS involved with the Environmental Assessment portion of the project continued groundwater monitoring during the last quarter. Wells were sampled in October and November. Water quality analyses are available for all samples collected through November. Tables 4.1 through 4.6 summarizes depths to water taken during the study. Tables 4.7 through 4.12 lists the cation and anion concentrations in the well samples as determined by the ISGS Geochemistry Laboratory. Well development by circulating potable water down the well casings just after installation of the monitoring wells has influenced initial groundwater quality. Water quality data, however, has stabilized. The water in the units bounding the target panels is a nonpotable brine.

The researchers also repaired an instrument station on the study site damaged by a truck collision and continued debugging the data logger programs to take reliable readings from the pressure transducers.

\section{$\underline{\text { Plans }}$}

Plans for the next quarter include continued monitoring of hydraulic head and groundwater chemistry at the study site. We will also attempt to sample shallower wells. 
Table 4.1

Screened interval and depth to water measurement in monitoring wells $2 \mathrm{~A}, 3$, and 4 .

Deepest wells in each nest are the sampled wells.

\begin{tabular}{|c|c|c|c|c|c|c|}
\hline Well nest & $2 \mathrm{~A}$ & $2 \mathrm{~A}$ & $2 \mathrm{~A}$ & 3 & 3 & 4 \\
\hline Screened interval (feet) & $75-80$ & $213.25-218.25$ & $340-345$ & $201-206$ & $282.5-287.5$ & $343.5-348.5$ \\
\hline $9 / 25 / 95^{*}$ & 16.87 & 83.7 & 237.45 & 27.5 & 13.17 & 257.73 \\
\hline $1 / 8 / 96$ & 21.22 & 82.46 & NW & 42.92 & 23.67 & - \\
\hline $2 / 6 / 96$ & 14.48 & 73.52 & NW & - & - & 341.43 \\
\hline $2 / 7 / 96$ & - & - & - & 44.51 & 274.21 & - \\
\hline $3 / 1 / 96$ & - & - & - & - & 279.18 & 341.77 \\
\hline $3 / 2 / 96$ & 12.9 & 72.5 & NW & - & - & - \\
\hline $3 / 11 / 96$ & 15.35 & 72.27 & NW & 47.36 & 282.8 & 344.16 \\
\hline $3 / 29 / 96$ & 14.37 & 71.54 & NW & 48.25 & 281.95 & 343.13 \\
\hline $4 / 9 / 96$ & 14.16 & 71.26 & NW & 48.83 & 282.81 & 343.91 \\
\hline $5 / 7 / 96$ & 14.46 & 70.33 & NW & 49.87 & 281.49 & 342.48 \\
\hline $5 / 15 / 96$ & 14.36 & 70.07 & NW & 49.97 & NW & 343.61 \\
\hline $6 / 10 / 96$ & - & - & - & 50.36 & 282.14 & 342.69 \\
\hline $6 / 11 / 96$ & 14.33 & 69.36 & NW & - & - & - \\
\hline $6 / 23 / 96$ & 14.09 & 69.25 & NW & 48.81 & NW & 343.44 \\
\hline $7 / 18 / 96$ & 14.65 & 68.90 & NA & 49.33 & NA & NA \\
\hline $7 / 26 / 96$ & 14.80 & 68.80 & NW & 49.51 & $\mathrm{NW}$ & 314.33 \\
\hline $8 / 23 / 96$ & 14.82 & 68.42 & NA & $* *$ & $* *$ & $\mathrm{NA}$ \\
\hline $8 / 30 / 96$ & 14.78 & 68.36 & $\mathrm{NA}$ & $51.95^{*}$ & $283.28^{*}$ & $\mathrm{NA}$ \\
\hline $10 / 2 / 96$ & 14.64 & 68.02 & NW & $* *$ & $* *$ & NW \\
\hline $10 / 12 / 96$ & 14.65 & 67.99 & NW & $* *$ & $* *$ & 344.99 \\
\hline $11 / 12 / 96$ & 14.605 & 67.755 & 345.72 & $* *$ & $* *$ & $\mathrm{NA}$ \\
\hline $11 / 21 / 96$ & 14.54 & 67.61 & NW & $* *$ & $* *$ & NA \\
\hline $1 / 7 / 96$ & 14.245 & 67.2 & 352.73 & 50.38 & 274.775 & 343.39 \\
\hline
\end{tabular}


Table 4.2

Screened interval and depth to water measurement in monitoring wells 5 and 6. Deepest wells in each nest are the sampled wells.

\begin{tabular}{|c|c|c|c|c|c|c|}
\hline Well nest & 5 & 5 & 5 & 6 & 6 & 6 \\
\hline Screened interval (feet) & $73-78$ & $215-220$ & $337-342$ & $71-76$ & $216.5-221.5$ & $311-316$ \\
\hline $9 / 25 / 95^{*}$ & 8.1 & 18.9 & 66.7 & 16.18 & 48.85 & 260.4 \\
\hline $1 / 8 / 96$ & - & - & - & - & - & - \\
\hline $2 / 6 / 96$ & 4.12 & 65.23 & N/A & 10.84 & 13.77 & NW \\
\hline $2 / 7 / 96$ & 4.56 & 83.94 & 313.44 & 13.4 & 21.47 & 312.77 \\
\hline $3 / 1 / 96$ & - & - & - & - & - & - \\
\hline $3 / 2 / 96$ & 3.38 & 55.13 & 326.4 & 11.1 & 25.8 & NW \\
\hline $3 / 11 / 96$ & N/A & N/A & $\mathrm{N} / \mathrm{A}$ & N/A & N/A & NW \\
\hline $3 / 29 / 96$ & 3.34 & 64.37 & NW & 10.79 & 27.67 & NW \\
\hline $4 / 9 / 96$ & 35.2 & 64.17 & $\mathrm{NW}$ & 10.28 & 28.29 & NW \\
\hline $5 / 7 / 96$ & N/A & N/A & N/A & N/A & N/A & N/A \\
\hline $5 / 15 / 96$ & 2.75 & 62.89 & 325.84 & 10.31 & 30.16 & $\mathrm{NW}$ \\
\hline $6 / 10 / 96$ & - & - & - & - & - & - \\
\hline $6 / 11 / 96$ & 3.86 & 64.66 & 325.24 & 10.18 & 31.82 & NW \\
\hline $6 / 23 / 96$ & 4.19 & 62.31 & 325.26 & 10.03 & 25.21 & $\mathrm{NW}$ \\
\hline $7 / 18 / 96$ & 4.31 & 62.93 & NA & 9.45 & 26.28 & NA \\
\hline $7 / 26 / 96$ & 4.38 & 42.33 & NW & 9.24 & 26.55 & NW \\
\hline $8 / 23 / 96$ & 3.96 & 63.36 & $\mathrm{NA}$ & 9.33 & 27.59 & NA \\
\hline $8 / 30 / 96$ & 4.30 & 62.87 & $\mathrm{NA}$ & $\mathrm{NA}$ & $\mathrm{NA}$ & NA \\
\hline $10 / 2 / 96$ & 4.34 & 64.33 & NW & 9.36 & 28.87 & NW \\
\hline $10 / 12 / 96$ & 5.93 & 63.32 & 327.44 & 9.62 & 29.12 & NW \\
\hline $11 / 12 / 96$ & 4.15 & 63.89 & NA & 9.56 & 29.975 & $316.2 ? ?$ \\
\hline $11 / 21 / 96$ & 4.545 & 63.75 & $\mathrm{NA}$ & 9.555 & 30.23 & NW \\
\hline $1 / 7 / 96$ & 4.35 & 62.36 & NW & 9.60 & 31.27 & 316.68 \\
\hline
\end{tabular}

N/A Indicates broken tape measure.

NW Indicates no measurable water level.

* Measuring tape not fully labeled so there is a greater, and unknown error with these readings.

No measurement taken on this date. 
Table 4.3

Depth to water measurement, samples collected, and flow-through-cell parameters for deep wells in each well nest, February 6 to March 11, 1996.

\begin{tabular}{|c|c|c|c|c|c|c|c|c|c|c|}
\hline Date & Well \# & DTW (feet) & $\begin{array}{c}\text { Time pumped } \\
\text { (min) }\end{array}$ & Cation sample & $\begin{array}{l}\text { Anion, Acidity, } \\
\text { Alkalinity sample }\end{array}$ & $\begin{array}{c}\text { Temperature } \\
\text { (C) }\end{array}$ & $\begin{array}{l}\text { ORP } \\
(\mathrm{mV})\end{array}$ & $\begin{array}{c}\text { Conductance } \\
\text { (mhos) }\end{array}$ & $\mathrm{pH}$ & $\mathrm{O} 2(\mathrm{ppm})$ \\
\hline $2 / 6 / 96$ & $2 \mathrm{~A}$ & NW & 38 & NW & NW & & & & & \\
\hline $2 / 7 / 96$ & 3 & 274.21 & 14 & 250 & 500,250 & $\overline{17.5}$ & 27 & 11.3 & 12.87 & 2.3 \\
\hline $2 / 6 / 96$ & 4 & 341.43 & 20 & 250 & IW (125) & 13.3 & 169 & 2.51 & 8.21 & 3.1 \\
\hline $2 / 7 / 96$ & 4 & ND & 150 & 250 & 500 & & & & & \\
\hline $2 / 6 / 96$ & 5 & NW & 14 & 250 & 500,250 & 15.7 & 56 & 17.2 & 12.88 & 4.3 \\
\hline $2 / 6 / 96$ & 6 & NW & 40 & NW & NW & & & & & \\
\hline $3 / 2 / 96$ & $2 \mathrm{~A}$ & NW & 20 & $\overline{N W}$ & $\overline{\mathrm{NW}}$ & & & & & \\
\hline $3 / 1 / 96$ & 3 & 279.18 & 25 & NW & IW (150) & 11.7 & -96 & 10 & 7.82 & 3.7 \\
\hline $3 / 2 / 96$ & 3 & ND & 65 & IW (75) & $\overline{\mathrm{NW}}$ & & & & & \\
\hline $3 / 1 / 96$ & 4 & 341.77 & 118 & NW & IW (25) & 9.7 & -78 & 5.03 & 7.24 & 2.3 \\
\hline $3 / 2 / 96$ & 4 & ND & 105 & 250 & IW (75) & & & & & \\
\hline $3 / 2 / 96$ & 5 & NW & 40 & NW & NW & & & & & \\
\hline $3 / 2 / 96$ & 6 & NW & 45 & NW & $\mathrm{NW}$ & & & & & \\
\hline $3 / 11 / 96$ & $2 \mathrm{~A}$ & NW & 18 & NW & NW & & & & & \\
\hline $3 / 11 / 96$ & 3 & 282.8 & 110 & 175 & 500 & & & & & \\
\hline $3 / 11 / 96$ & 3 & ND & 71 & 175 & NS & & & & & \\
\hline $3 / 11 / 96$ & 4 & 344.16 & 10 & 250 & 500 & 8.4 & 19 & 4.99 & 8.47 & 7.1 \\
\hline $3 / 11 / 96$ & 5 & ND & 20 & IW & IW (25) & & & & & \\
\hline $3 / 11 / 96$ & 6 & NW & 15 & $\mathrm{NW}$ & $\overline{\mathrm{NW}}$ & & & & & \\
\hline
\end{tabular}


Table 4.4

Depth to water measurement, samples collected, and flow-through-cell parameters for deep wells in each well nest, March 29 to May 15, 1996.

\begin{tabular}{|c|c|c|c|c|c|c|c|c|c|c|}
\hline Date & Well \# & DTW (feet) & $\begin{array}{l}\text { Time pumped } \\
\text { (min) }\end{array}$ & Cation sample & $\begin{array}{c}\text { Anion Acidity } \\
\text { Alkalinity sample }\end{array}$ & \begin{tabular}{|c|} 
Temperature \\
(C)
\end{tabular} & $\begin{array}{l}\text { ORP } \\
(\mathrm{mV})\end{array}$ & $\begin{array}{c}\text { Conductance } \\
\text { (mhos) }\end{array}$ & $\mathrm{pH}$ & $\mathrm{O} 2(\mathrm{ppm})$ \\
\hline $3 / 29 / 96$ & $2 \mathrm{~A}$ & NW & 13 & NS & NS & & & & & \\
\hline $3 / 29 / 96$ & 3 & 281.95 & 57 & NS & NS & 11.3 & -52 & 12.6 & 7.24 & 7.3 \\
\hline $3 / 29 / 96$ & 4 & 343.13 & 82 & $\mathrm{NS}$ & $\mathrm{NS}$ & 9.6 & -44 & 7.25 & 6.96 & 3.4 \\
\hline $3 / 29 / 96$ & 5 & NW & 20 & NS & $\mathrm{NS}$ & & & & & \\
\hline $3 / 29 / 96$ & 6 & NW & & & & & & & & \\
\hline $4 / 9 / 96$ & $2 \mathrm{~A}$ & NW & 10 & NS & NS & & & & & \\
\hline $4 / 9 / 96$ & 3 & 282.81 & 118 & 230 & 500 & & & & & \\
\hline $4 / 9 / 96$ & 4 & 343.91 & 26 & 250 & 500 & & & & & \\
\hline $4 / 9 / 96$ & 5 & NW & 18 & IW & IW (20) & & & & & \\
\hline $4 / 9 / 96$ & 6 & NW & 11 & NW & NW & & & & & \\
\hline $5 / 15 / 96$ & $2 \mathrm{~A}$ & NW & 11 & NS & NS & & & & & \\
\hline $5 / 15 / 96$ & 3 & NW & 103 & IW (50-75) & 500 & & & & & \\
\hline $5 / 15 / 96$ & 4 & 343.61 & 7 & 250 & 500 & 20.2 & 40 & 12.1 & 8.14 & 3.3 \\
\hline $5 / 15 / 96$ & 5 & 325.84 & 15 & NS & NS & & & & & \\
\hline $5 / 15 / 96$ & 6 & NW & * & * & & & & & & \\
\hline
\end{tabular}


Table 4.5

Depth to water measurement, samples collected, and flow-through-cell parameters for deep wells in each well nest, June 23 to October 12, 1996.

\begin{tabular}{|c|c|c|c|c|c|c|c|c|c|c|}
\hline Datc & Well \# & DTW (feet) & $\begin{array}{l}\text { Time pumped } \\
\text { (min) }\end{array}$ & Cation sample & $\begin{array}{c}\text { Anion Acidity } \\
\text { Alkalinity sample }\end{array}$ & $\begin{array}{l}\text { Temperature } \\
\text { (C) }\end{array}$ & $\begin{array}{l}\text { ORP } \\
(\mathrm{mV})\end{array}$ & $\begin{array}{c}\text { Conductance } \\
\text { (mhos) }\end{array}$ & $\mathrm{pH}$ & $\mathrm{O} 2(\mathrm{ppm})$ \\
\hline $6 / 23 / 96$ & $2 \mathrm{~A}$ & NW & 10 & NS & NS & & & & & \\
\hline $6 / 23 / 96$ & 3 & NW & 112 & IW (100) & IW (450) & & & & & \\
\hline $6 / 23 / 96$ & 4 & 343.44 & 10 & 250 & 500 & 23.8 & -75 & 15.8 & 7.87 & 3.4 \\
\hline $6 / 23 / 96$ & 5 & 325.26 & 23 & 250 & 500 & & & & & \\
\hline $6 / 23 / 96$ & 6 & NW & 10 & & & & & & & \\
\hline $7 / 26 / 96$ & $2 \mathrm{~A}$ & NW & 10 & NS & NS & & & & & \\
\hline $7 / 26 / 96$ & 3 & NW & 60 & NS & IW-(250) & & & & & \\
\hline $7 / 26 / 96$ & 4 & 314.33 & 12 & 250 & 500 & 33.9 & 45 & 16.1 & 8.19 & 2.7 \\
\hline $7 / 26 / 96$ & 5 & NW & 8 & NS & $\mathrm{NS}$ & & & & & \\
\hline $7 / 26 / 96$ & 6 & NW & 20 & $\overline{\mathrm{NS}}$ & NS & & & & & \\
\hline $8 / 30 / 96$ & $2 \mathrm{~A}$ & NA & 15 & NS & NS & & & & & \\
\hline $8 / 30 / 96$ & 3 & 283.28 & $0^{* * *}$ & $* *$ & $* *$ & & & & & \\
\hline $8 / 30 / 96$ & 4 & NA & 26 & 250 & 500 & & & & & \\
\hline $8 / 30 / 96$ & 5 & NA & 10 & NS & NS & & & & & \\
\hline $8 / 30 / 96$ & 6 & NA & NA & NA & NA & & & & & \\
\hline $10 / 12 / 96$ & $2 \mathrm{~A}$ & NW & 12 & NS & NS & & & & & \\
\hline $10 / 12 / 96$ & 4 & 344.99 & 77 & 250 & 500 & & & & & \\
\hline $10 / 12 / 96$ & 5 & 327.44 & 8 & NS & NS & & & & & \\
\hline $10 / 12 / 96$ & 6 & NW & 10 & NS & NS & & & & & \\
\hline
\end{tabular}


Table 4.6

Depth to water measurement, samples collected, and flow-through-cell parameters for deep wells in each well nest,

November 12, 1996 to January 7, 1997

\begin{tabular}{|c|c|c|c|c|c|c|c|c|c|c|}
\hline Date & Well \# & DTW (fect) & $\begin{array}{c}\text { Time pumped } \\
\text { (min) }\end{array}$ & Cation sample & $\begin{array}{c}\text { Anion Acidity } \\
\text { Alkalinity sample }\end{array}$ & $\begin{array}{c}\text { Temperature } \\
\text { (C) } \\
\end{array}$ & $\begin{array}{l}\text { ORP } \\
(\mathrm{mV})\end{array}$ & $\begin{array}{c}\text { Conductance } \\
\text { (mhos) }\end{array}$ & $\mathrm{pH}$ & $\mathrm{O} 2(\mathrm{ppm})$ \\
\hline $11 / 12 / 96$ & $2 \mathrm{~A}$ & 345.72 & 10 & & & & & & & \\
\hline $11 / 12 / 96$ & 3 & $\mathrm{NA}$ & $* *$ & & & & & & & \\
\hline $11 / 12 / 96$ & 4 & $\mathrm{NA}$ & 15 & & & & & & & \\
\hline $11 / 12 / 96$ & 5 & $\overline{N A}$ & 12 & & & & & & & \\
\hline $11 / 12 / 96$ & 6 & $316.2 ?$ & 10 & & & & & & & \\
\hline $11 / 21 / 96$ & $2 \mathrm{~A}$ & NW & 11 & & & & & & & \\
\hline $11 / 21 / 96$ & 3 & $\mathrm{NA}$ & $* *$ & & & & & & & \\
\hline $11 / 21 / 96$ & 4 & $\mathrm{NA}$ & 95 & 500 & 250 & & & & & \\
\hline $11 / 21 / 96$ & 5 & $\mathrm{NA}$ & 10 & & & & & & & \\
\hline $11 / 21 / 96$ & 6 & NW & * & & & & & & & \\
\hline $1 / 7 / 97$ & $2 \mathrm{~A}$ & 352.73 & 10 & & & & & & & \\
\hline $1 / 7 / 97$ & 3 & 274.78 & 20 & & & & & & & \\
\hline $1 / 7 / 97$ & 4 & 343.39 & 35 & & & 8.0 & 76 & 14.6 & 7.46 & 6.4 \\
\hline $1 / 7 / 97$ & 5 & NW & 10 & & & & & & & \\
\hline $1 / 7 / 97$ & 6 & 316.68 & 10 & & & & & & & \\
\hline
\end{tabular}

IW Water yielded but insufficient for sample. Amount collected in parentheses.

NA Electric tape measure not functioning

ND No depth to water measurement taken.

NS No water available for sampling.

NW No measurable water in well.

* Could not access well with sampling equipment.

** Well damaged and unpumpable 
Table 4.7

Laboratory analysis of cation constituents, February and March sampling, 1996. All units are mg/L, P unless noted.

\begin{tabular}{|c|c|c|c|c|c|c|c|c|c|c|c|c|}
\hline Lab \# & Date & Well \# & $\mathrm{Al}$ & As & $B$ & $\mathrm{Ba}$ & $\mathrm{Be}^{*}$ & $\mathrm{Ca}$ & $\mathrm{Cd}$ & $\mathrm{CO}$ & $\mathrm{CR}$ & $\mathrm{CU}$ \\
\hline W01003 & $2 / 6 / 96$ & 4 & $<0.02$ & $<0.1$ & 0.42 & 0.14 & $<2$ & 39.7 & $<0.01$ & $<0.01$ & $<0.01$ & $<0.01$ \\
\hline W01005 & $2 / 6 / 96$ & 5 & 0.29 & $<0.1$ & 0.42 & 1.73 & $<2$ & 115 & $<0.01$ & $<0.01$ & $<0.01$ & $<0.01$ \\
\hline W01006 & $2 / 7 / 96$ & 3 & 0.11 & $<0.1$ & 0.54 & 0.37 & $<2$ & 10.1 & $<0.01$ & $<0.01$ & $<0.01$ & $<0.01$ \\
\hline W01004 & $2 / 7 / 96$ & 4 & $<0.02$ & $<0.1$ & 0.4 & 0.12 & $<2$ & 38.8 & $<0.01$ & $<0.01$ & $<0.01$ & $<0.01$ \\
\hline W01007 & $2 / 7 / 96$ & blank & $<0.02$ & $<0.1$ & $<0.02$ & $<0.02$ & $<2$ & $<0.01$ & $<0.01$ & $<0.01$ & $<0.01$ & $<0.01$ \\
\hline W01063 & $3 / 10 / 96$ & 3 & 0.1 & $<0.1$ & 0.77 & 0.19 & $<2$ & 14.8 & $<0.01$ & $<0.01$ & $<0.01$ & $<0.01$ \\
\hline W01064 & $3 / 10 / 96$ & 4 & 0.03 & $<0.1$ & 0.51 & 0.14 & $<2$ & 67.8 & $<0.01$ & $<0.01$ & $<0.01$ & $<0.01$ \\
\hline W01065 & $3 / 10 / 96$ & blank & $<0.01$ & $<0.1$ & $<0.02$ & $<0.01$ & $<2$ & $<0.01$ & $<0.01$ & $<0.01$ & $<0.01$ & $<0.01$ \\
\hline Lab \# & Date & Well \# & FE & $\mathrm{K}$ & $\mathrm{LA}^{*}$ & LI & MG & MN & MO & NA & $\mathrm{NI}$ & PB \\
\hline W01003 & $2 / 6 / 96$ & 4 & 0.02 & 7 & $<2$ & 0.03 & 12 & 0.09 & 0.03 & 377 & $<0.03$ & $<0.05$ \\
\hline W01005 & $2 / 6 / 96$ & 5 & 0.01 & 224 & $<2$ & 1.51 & 0.01 & $<0.01$ & 0.12 & 1605 & $<0.03$ & $<0.05$ \\
\hline W01006 & $2 / 7 / 96$ & 3 & 0.01 & 418 & $<2$ & 1.22 & 0.02 & $<0.01$ & 0.13 & 1027 & $<0.03$ & $<0.05$ \\
\hline W01004 & $2 / 7 / 96$ & 4 & 0.01 & 8 & $<2$ & 0.03 & 12 & 0.08 & 0.02 & 374 & $<0.03$ & $<0.05$ \\
\hline W01007 & $2 / 7 / 96$ & blank & $<0.01$ & $<1$ & $<2$ & $<0.01$ & $<0.01$ & $<0.01$ & $<0.02$ & $<0.1$ & $<0.03$ & $<0.05$ \\
\hline W01063 & $3 / 10 / 96$ & 3 & 0.01 & 324 & $<2$ & 1.02 & 0.01 & $<0.01$ & 0.11 & 1680 & $<0.03$ & $<0.08$ \\
\hline W01064 & $3 / 10 / 96$ & 4 & 0.04 & 12 & $<2$ & 0.05 & 20.2 & $<0.01$ & $<0.02$ & 705 & $<0.03$ & $<0.08$ \\
\hline W01065 & $3 / 10 / 96$ & blank & $<0.01$ & $<1$ & $<2$ & $<0.01$ & $<0.01$ & $<0.01$ & $<0.02$ & 0.6 & $<0.03$ & $<0.08$ \\
\hline Lab \# & Date & Well \# & SB & $\mathrm{SC}^{*}$ & $\overline{S E}$ & SI & SR & $\mathrm{TI}$ & $\mathrm{TL}$ & $\mathrm{V}$ & $\mathrm{ZN}$ & ZR \\
\hline W01003 & $2 / 6 / 96$ & 4 & $<0.2$ & $<3$ & $<0.1$ & 1.79 & 0.62 & $<0.01$ & $<0.6$ & $<0.01$ & 0.01 & $<0.01$ \\
\hline W01005 & $2 / 6 / 96$ & 5 & $<0.2$ & $<3$ & $<0.1$ & 1.77 & 8.68 & $<0.01$ & $<0.6$ & $<0.01$ & 0.3 & $<0.01$ \\
\hline W01006 & $2 / 7 / 96$ & 3 & $<0.2$ & $<3$ & $<0.1$ & 1.79 & 1.54 & $<0.01$ & $<0.6$ & $<0.01$ & 0.01 & $<0.01$ \\
\hline W01004 & $2 / 7 / 96$ & 4 & $<0.2$ & $<3$ & $<0.1$ & 1.82 & 0.6 & $<0.01$ & $<0.6$ & $<0.01$ & 0.02 & $<0.01$ \\
\hline W01007 & $2 / 7 / 96$ & blank & $<0.2$ & $<3$ & $<0.1$ & $<0.01$ & $<0.01$ & $<0.01$ & $<0.6$ & $<0.01$ & $<0.01$ & $<0.01$ \\
\hline W01063 & $3 / 10 / 96$ & 3 & $<0.1$ & $<3$ & $<0.1$ & 2.11 & 1.86 & $<0.01$ & $<0.3$ & $<0.01$ & 0.01 & $<0.01$ \\
\hline W01064 & $3 / 10 / 96$ & 4 & $<0.1$ & $<3$ & $<0.1$ & 2.13 & 1.31 & $<0.01$ & $<0.3$ & $<0.01$ & 0.01 & $<0.01$ \\
\hline W01065 & $3 / 10 / 96$ & blank & $<0.1$ & $<3$ & $<0.1$ & $<0.01$ & $<0.01$ & $<0.01$ & $<0.3$ & $<0.01$ & $<0.01$ & $<0.01$ \\
\hline
\end{tabular}


Table 4.8

Laboratory analysis of anion constituents, February and March sampling, 1996. . All units are mg/L, P unless noted.

\begin{tabular}{||l|c|c|c|c|c|c|c|c|c|c||}
\hline Lab \# & Date & Well \# & HG $*$ & CL & $\mathrm{F}$ & NO3 $* *$ & SO4** & Total K $* * *$ & Pal K *** & Acid *** \\
\hline W01003 & $2 / 6 / 96$ & 4 & $<0.05$ & ND & ND & ND & ND & 93.5 & ND & 4 \\
\hline W01005 & $2 / 6 / 96$ & 5 & $<0.05$ & 1064 & ND & 0.09 & 81.9 & 2532 & 2418 & ND \\
\hline W01006 & $2 / 7 / 96$ & 3 & $<0.05$ & 705 & ND & 0.19 & 250 & 901.3 & 823.1 & ND \\
\hline W01004 & $2 / 7 / 96$ & 4 & $<0.05$ & 478 & ND & 0.1 & 214 & 127.6 & ND & 3 \\
\hline W01007 & $2 / 7 / 96$ & blank & $<0.05$ & ND & ND & ND & ND & ND & ND & ND \\
\hline W01063 & $3 / 10 / 96$ & 3 & $<0.05$ & 1668 & ND & 0.07 & 549 & 1033 & 953 & ND \\
\hline W01064 & $3 / 10 / 96$ & 4 & $<0.05$ & 1113 & ND & 0.08 & 291 & 150.9 & ND & 5 \\
\hline W01065 & $3 / 10 / 96$ & blank & 0.07 & 2.14 & ND & $<0.01$ & 0.31 & 1 & ND & 2 \\
\hline
\end{tabular}

Remarks:

Total $\mathrm{K} \quad$ Alkalinity to $\mathrm{pH} 4.5$ as $\mathrm{mg} \mathrm{CaCO}_{3} / \mathrm{L}$

Pal K Alkalinity to $\mathrm{pH} 8.3 \mathrm{as} \mathrm{mg} \mathrm{CaCO}_{3} / \mathrm{L}$

Acid Acidity to $\mathrm{pH} 8.3 \mathrm{as} \mathrm{mg} \mathrm{CaCO} / \mathrm{L}$

Analytic Method Codes:

* $\quad \mu \mathrm{g} / \mathrm{L}$, Inductively coupled plasma

** $\quad \mathrm{mg} / \mathrm{L}$, Ion chromatography

*** $\quad \mathrm{mg} / \mathrm{L}$, Titrimetric

ND No detection 
Table 4.9

Laboratory analysis of cation constituents, April, May, and June sampling, 1996. All units are mg/L, P unless noted.

\begin{tabular}{|c|c|c|c|c|c|c|c|c|c|c|c|c|}
\hline Lab\# & Date & Well \# & $\mathrm{Al}$ & As & B & $\mathrm{Ba}$ & $\mathrm{Be}^{*}$ & $\mathrm{Ca}$ & $\mathrm{Cd}$ & $\mathrm{CO}$ & $\mathrm{CR}$ & $\mathrm{CU}$ \\
\hline W01122 & $4 / 9 / 96$ & 3 & 0.1 & $<0.1$ & 0.77 & 0.14 & $<1$ & 20.2 & $<0.01$ & $<0.01$ & $<0.1$ & $<0.01$ \\
\hline W01123 & $4 / 9 / 96$ & 4 & 0.04 & $<0.1$ & 0.58 & 0.14 & $<1$ & 97.6 & $<0.01$ & $<0.01$ & $<0.1$ & $<0.01$ \\
\hline W01229 & $5 / 15 / 96$ & 3 & 0.04 & $<0.1$ & 0.78 & 0.13 & $<1$ & 19.4 & $<0.1$ & $<0.1$ & $<0.1$ & 0.01 \\
\hline W01228 & $5 / 15 / 96$ & 4 & $<.02$ & $<0.1$ & 0.66 & 0.15 & 1 & 118 & $<0.1$ & $<0.1$ & $<0.1$ & 0.01 \\
\hline W01230 & $5 / 15 / 96$ & blank & $<.02$ & $<0.1$ & $<0.01$ & $<0.01$ & $<1$ & 0.02 & $<0.1$ & $<0.1$ & $<0.1$ & $<0.01$ \\
\hline W01363 & $6 / 23 / 96$ & 3 & 0.05 & $<0.1$ & 0.78 & 0.13 & 1 & 23 & $<0.1$ & $<0.1$ & $<0.1$ & $<0.01$ \\
\hline W01362 & $6 / 23 / 96$ & 4 & 0.04 & $<0.1$ & 0.82 & 0.16 & 2 & 168 & $<0.1$ & $<0.1$ & $<0.1$ & 0.01 \\
\hline W01364 & $6 / 23 / 96$ & 5 & 0.24 & $<0.1$ & 0.27 & 4.78 & 2 & 328 & $<0.1$ & $<0.1$ & $<0.1$ & $<0.01$ \\
\hline W01365 & $6 / 23 / 96$ & blank & $<.02$ & $<0.1$ & $<0.02$ & $<0.01$ & $<1$ & 0.06 & $<0.1$ & $<0.1$ & $<0.1$ & $<0.01$ \\
\hline Lab \# & Date & Well \# & FE & $\mathrm{K}$ & $\mathrm{LA}^{*}$ & $\mathrm{LI}$ & $\mathrm{MG}$ & $\mathrm{MN}$ & $\mathrm{MO}$ & NA & $\mathrm{NI}$ & PB \\
\hline W01122 & $4 / 9 / 96$ & 3 & 0.03 & 255 & $<2$ & 0.88 & 0.03 & $<0.01$ & 0.12 & 1780 & $<0.03$ & $<0.05$ \\
\hline W01123 & $4 / 9 / 96$ & 4 & 0.09 & 12 & $<2$ & 0.06 & 30 & 0.03 & $<0.02$ & 1030 & $<0.03$ & $<0.05$ \\
\hline W01229 & $5 / 15 / 96$ & 3 & $<0.01$ & 229 & $<2$ & 0.81 & 0.02 & $<0.01$ & 0.12 & 1830 & $<0.3$ & $<0.5$ \\
\hline W01228 & $5 / 15 / 96$ & 4 & 0.07 & 14 & $<2$ & 0.07 & 36.7 & 0.03 & $<0.02$ & 1260 & $<0.3$ & $<0.5$ \\
\hline W01230 & $5 / 15 / 96$ & blank & $<0.01$ & $<1$ & $<2$ & $<0.01$ & $<0.01$ & $<0.01$ & $<0.02$ & 1.1 & $<0.3$ & $<0.5$ \\
\hline W01363 & $6 / 23 / 96$ & 3 & 0.04 & 185 & $<2$ & 0.66 & 0.12 & $<0.01$ & 0.1 & 2280 & $<0.02$ & $<0.08$ \\
\hline W01362 & $6 / 23 / 96$ & 4 & 0.15 & 19 & $<2$ & 0.09 & 52.7 & 0.04 & $<0.02$ & 2040 & $<0.02$ & $<0.08$ \\
\hline W01364 & $6 / 23 / 96$ & 5 & 0.02 & 159 & $<2$ & 1.07 & 0.01 & $<0.01$ & 0.15 & 4100 & $<0.2$ & $<0.8$ \\
\hline W01365 & $6 / 23 / 96$ & blank & 0.02 & $<1$ & $<2$ & $<0.01$ & $<0.01$ & $<0.01$ & $<0.02$ & 0.36 & $<0.2$ & $<0.8$ \\
\hline Lab \# & Date & Well \# & SB & $\mathrm{SC}^{*}$ & SE & SI & SR & $\mathrm{TI}$ & $\mathrm{TL}$ & $\mathrm{V}$ & $\mathrm{ZN}$ & $\mathrm{ZR}$ \\
\hline W01122 & $4 / 9 / 96$ & 3 & $<0.1$ & $<3$ & $<0.1$ & 1.81 & 1.9 & $<0.01$ & $<0.3$ & $<0.01$ & $<0.01$ & $<0.01$ \\
\hline W01123 & $4 / 9 / 96$ & 4 & $<0.1$ & $<3$ & $<0.1$ & 2.46 & 2.1 & $<0.01$ & $<0.3$ & $<0.01$ & 0.01 & $<0.01$ \\
\hline W01229 & $5 / 15 / 96$ & 3 & $<0.1$ & $<3$ & $<0.2$ & 1.68 & 1.97 & $<0.1$ & $<0.3$ & $<0.1$ & $<0.01$ & $<0.1$ \\
\hline W01228 & $5 / 15 / 96$ & 4 & $<0.1$ & $<3$ & $<0.2$ & 2.7 & 2.79 & $<0.1$ & 0.4 & $<0.1$ & 0.01 & $<0.1$ \\
\hline W01230 & $5 / 15 / 96$ & blank & $<0.1$ & $<3$ & $<0.2$ & $<0.01$ & $<0.01$ & $<0.1$ & $<0.3$ & $<0.1$ & $<0.01$ & $<0.1$ \\
\hline W01363 & $6 / 23 / 96$ & 3 & $<0.2$ & $<3$ & $<0.2$ & 1.59 & 2.25 & $<0.1$ & $<0.3$ & $<0.1$ & $<0.01$ & $<0.1$ \\
\hline W01362 & $6 / 23 / 96$ & 4 & $<0.2$ & $<3$ & $<0.2$ & 3.12 & 4.01 & $<0.1$ & $<0.3$ & $<0.1$ & 0.02 & $<0.1$ \\
\hline W01364 & $6 / 23 / 96$ & 5 & $<0.2$ & $<3$ & $<0.2$ & 0.68 & 12.75 & $<0.1$ & $<0.3$ & $<0.1$ & 0.02 & $<0.1$ \\
\hline W01365 & $6 / 23 / 96$ & blank & $<0.2$ & $<3$ & $<0.2$ & $<0.01$ & 0.01 & $<0.1$ & $<0.3$ & $<0.1$ & 0.03 & $<0.1$ \\
\hline
\end{tabular}


Table 4.10

Laboratory analysis of anion constituents, April, May, and June sampling, 1996. All units are mg/L, P unless noted.

\begin{tabular}{|c|c|c|c|c|c|c|c|c|c|c|}
\hline Lab \# & Date & Well \# & $\mathrm{HG} *$ & $\mathrm{CL}$ & $\mathrm{F}$ & $\mathrm{NO} 3 * *$ & $\mathrm{SO} 4 * *$ & Total $\mathrm{K} * * *$ & Pal K *** & Acid *** \\
\hline W01122 & $4 / 9 / 96$ & 3 & 0.31 & 2059 & ND & 0.04 & 684 & 833.6 & 789.4 & ND \\
\hline W01123 & $4 / 9 / 96$ & 4 & 0.34 & 1527 & $\mathrm{ND}$ & 0.06 & 383 & 162.2 & ND & 7 \\
\hline W01229 & $5 / 15 / 96$ & 3 & 0.06 & 2614 & ND & $<0.01$ & 682 & 629.9 & 597.7 & $\mathrm{ND}$ \\
\hline W01228 & $5 / 15 / 96$ & 4 & 0.09 & 2062 & ND & $<0.01$ & 327 & 171.3 & ND & 9 \\
\hline W01230 & $5 / 15 / 96$ & blank & 0.09 & $<0.01$ & ND & $<0.01$ & $<0.01$ & 1.8 & $\mathrm{ND}$ & ND \\
\hline W01363 & $6 / 23 / 96$ & 3 & 0.08 & 2994 & ND & 0.39 & 745 & 382.6 & 367.5 & ND \\
\hline W01362 & $6 / 23 / 96$ & 4 & $<0.05$ & 3385 & ND & 0.44 & 401 & 205.5 & ND & 13 \\
\hline Wo1364 & $6 / 23 / 96$ & 5 & $<0.05$ & 6045 & ND & $<0.01$ & 26.8 & 2804 & 2760 & ND \\
\hline W01365 & $6 / 23 / 96$ & blank & $<0.05$ & 0.29 & ND & $<0.01$ & $<0.01$ & 0.9 & ND & I \\
\hline
\end{tabular}

Remarks:

Total $\mathrm{K}$ Alkalinity to $\mathrm{pH} 4.5$ as $\mathrm{mg} \mathrm{CaCO}_{2} / \mathrm{L}$.

Pal K Alkalinity to $\mathrm{pH} 8.3$ as $\mathrm{mg} \mathrm{CaCO}_{3} / \mathrm{L}$

Acid Acidity to $\mathrm{pH} 8.3$ as $\mathrm{mg} \mathrm{CaCO}_{3} / \mathrm{L}$

Analytic Method Codes:

* $\quad \mu \mathrm{g} / \mathrm{L}$, Inductively coupled plasma

** $\quad \mathrm{mg} / \mathrm{L}$, Ion chromatography

*** $\quad \mathrm{mg} / \mathrm{L}$, Titrimetric

ND No detection 
Table 4.11

Laboratory analysis of cation constituents, July through November sampling, 1996. All units are mg/L, P unless noted.

\begin{tabular}{|c|c|c|c|c|c|c|c|c|c|c|c|c|}
\hline Lali & Dalc & Well \# & $\mathrm{Al}$ & As & $B$ & $\mathrm{Ba}$ & $\mathrm{Be} *$ & $\mathrm{Ca}$ & $\mathrm{Cd}$ & $\mathrm{CO}$ & $\mathrm{CR}$ & $\mathrm{CU}$ \\
\hline W01497 & $7 / 26 / 96$ & 3 & ND & ND & ND & ND & ND & ND & ND & ND & ND & ND \\
\hline W01496 & $7 / 26 / 96$ & 4 & $<0.02$ & $<0.1$ & 0.86 & 0.14 & 1 & 176 & $<0.01$ & $<0.01$ & $<0.01$ & $<0.01$ \\
\hline W01498 & $7 / 26 / 96$ & blank & $<0.02$ & $<0.1$ & $<0.01$ & $<0.01$ & $<1$ & 0.07 & $<0.01$ & $<0.01$ & $<0.01$ & $<0.01$ \\
\hline W01585 & $8 / 30 / 96$ & 4 & 0.04 & $<0.1$ & 1.00 & 0.15 & 3 & 200 & $<0.01$ & $<0.01$ & $<0.01$ & $<0.01$ \\
\hline W01586 & $8 / 30 / 96$ & blank & $<0.01$ & $<0.1$ & $<0.02$ & $<0.01$ & $<1$ & 0.01 & $<0.01$ & $<0.01$ & $<0.01$ & $<0.01$ \\
\hline W01771 & $10 / 12 / 96$ & 4 & 0.06 & $<0.1$ & 0.89 & 0.12 & $<1$ & 166 & $<0.01$ & $<0.01$ & $<0.01$ & 0.01 \\
\hline W01772 & $10 / 12 / 96$ & blank & $<0.03$ & $<0.1$ & & $<0.01$ & $<1$ & 0.01 & $<0.01$ & $<0.01$ & $<0.01$ & $<0.01$ \\
\hline W01867 & $11 / 21 / 96$ & 4 & $<0.03$ & $<0.1$ & 0.99 & 0.12 & $<1$ & 186 & $<0.01$ & $<0.01$ & $<0.01$ & 0.01 \\
\hline \begin{tabular}{|l|} 
W01868 \\
\end{tabular} & $11 / 21 / 96$ & blank & $<0.03$ & $<0.1$ & $<0.01$ & $<0 / 01$ & $<1$ & 0.03 & $<0.01$ & $<0.01$ & $<0.01$ & $<0.01$ \\
\hline Lab \# & Date & Well \# & FE & $\mathrm{K}$ & $\mathrm{LA}^{*}$ & LI & MG & $\mathrm{MN}$ & $\mathrm{MO}$ & NA & $\mathrm{NI}$ & $\mathrm{PB}$ \\
\hline W01497 & $7 / 26 / 96$ & 3 & ND & ND & $\mathrm{ND}$ & ND & ND & $\mathrm{ND}$ & ND & ND & ND & ND \\
\hline W01496 & $7 / 26 / 96$ & 4 & 0.11 & 20 & $<2$ & 0.09 & 56.9 & 0.07 & $<0.02$ & 2290 & $<0.03$ & $<0.04$ \\
\hline W01498 & $7 / 26 / 96$ & blank & $<0.01$ & $<1$ & $<2$ & $<0.01$ & 0.02 & $<0.01$ & $<0.02$ & 1 & $<0.03$ & $<0.04$ \\
\hline W01585 & $8 / 30 / 96$ & 4 & 0.10 & 22 & $<2$ & 0.09 & 63.5 & 0.10 & $<0.02$ & 2770 & 0.05 & $<0.05$ \\
\hline W01586 & $8 / 30 / 96$ & blank & $<0.01$ & $<1$ & $<2$ & $<0.03$ & 0.01 & $<0.01$ & $<0.02$ & 1 & $<0.03$ & $<0.05$ \\
\hline W01771 & $10 / 12 / 96$ & 4 & $<0.01$ & 22 & $<2$ & 0.10 & 53.9 & 0.10 & $<0.02$ & 2510 & 0.05 & $<0.05$ \\
\hline W01772 & $10 / 12 / 96$ & blank & $<0.01$ & 3 & $<2$ & $<0.01$ & 0.01 & $<0.01$ & $<0.02$ & 0.40 & $<0.03$ & $<0.05$ \\
\hline W01867 & $11 / 21 / 96$ & 4 & $<0.01$ & 21 & $<2$ & 0.12 & 57.5 & 0.15 & $<0.02$ & 2790 & $<0.03$ & $<0.05$ \\
\hline W01868 & $11 / 21 / 96$ & blank & $<0.01$ & $<1$ & $<2$ & $<0.01$ & 0.01 & $<0.01$ & $<0.02$ & $<0.03$ & $<0.03$ & $<0.05$ \\
\hline Lab \# & Date & Well \# & SB & $\mathrm{SC}^{*}$ & SE & SI & SR & $\mathrm{TI}$ & TL & $\mathrm{V}$ & $\mathrm{ZN}$ & $\overline{Z R}$ \\
\hline W01497 & $7 / 26 / 96$ & 3 & ND & ND & ND & ND & ND & $\mathrm{ND}$ & $\mathrm{ND}$ & ND & ND & $\mathrm{ND}$ \\
\hline W01496 & $7 / 26 / 96$ & 4 & $<0.2$ & $<3$ & $<0.2$ & 3.16 & 4.40 & $<0.01$ & $<0.2$ & $<0.01$ & 0.01 & $<0.01$ \\
\hline W01498 & $7 / 26 / 96$ & blank & $<0.2$ & $<3$ & $<0.2$ & $<0.01$ & $<0.01$ & $<0.01$ & $<0.2$ & $<0.01$ & $<0.01$ & $<0.01$ \\
\hline W01585 & $8 / 30 / 96$ & 4 & $<0.2$ & $<7$ & $<0.2$ & 3.52 & 5.11 & $<0.01$ & $<0.3$ & $<0.01$ & 0.02 & $<0.01$ \\
\hline W01586 & $8 / 30 / 96$ & blank & $<0.2$ & $<7$ & $<0.2$ & $<0.01$ & $<0.01$ & $<0.01$ & $<0.3$ & $<0.01$ & $<0.01$ & $<0.01$ \\
\hline W01771 & $10 / 12 / 96$ & 4 & $<0.2$ & $<3$ & $<0.2$ & 3.21 & 4.44 & $<0.01$ & $<0.3$ & $<0.01$ & 0.04 & $<0.01$ \\
\hline W01772 & $10 / 12 / 96$ & blank & $<0.2$ & $<3$ & $<0.2$ & $<0.01$ & $<0.01$ & $<0.01$ & $<0.3$ & $<0.01$ & $<0.01$ & $<0.01$ \\
\hline W01867 & $11 / 21 / 96$ & 4 & $<0.2$ & $<3$ & $<0.2$ & 3.4 & 4.79 & $<0.01$ & $<0.3$ & $<0.01$ & 0.02 & $<0.01$ \\
\hline W01868 & $11 / 21 / 96$ & blank & $<0.2$ & $<3$ & $<0.2$ & $<0.01$ & $<0.01$ & $<0.01$ & $<0.3$ & $<0.01$ & $<0.01$ & $<0.01$ \\
\hline
\end{tabular}


Table 4.12

Laboratory analysis of anion constituents, July through November sampling, 1996. All units are mg/L, P unless noted.

\begin{tabular}{|c|c|c|c|c|c|c|c|c|c|c|c|}
\hline Date & Well \# & HG $*$ & CL & $\mathrm{F}$ & NO3 $* *$ & SO $*^{* *}$ & Total K *** & Pal K *** & Acid *** & TDS \\
\hline $7 / 26 / 96$ & 3 & & & & & & 210.5 & 205.8 & 6 & \\
\hline $7 / 26 / 96$ & 4 & & & & & & 226.9 & ND & 7 & \\
\hline $7 / 26 / 96$ & blank & & & & & & 2 & ND & ND & \\
\hline $8 / 30 / 96$ & 4 & & & & & & 239.2 & ND & 8 & \\
\hline $8 / 30 / 96$ & blank & & & & & & $<2$ & ND & ND & \\
\hline $10 / 12 / 96$ & 4 & & & & & & 247.6 & ND & 19 & 8204 \\
\hline $10 / 12 / 96$ & blank & & & & & & $<1$ & ND & ND & $<10$ \\
\hline $11 / 21 / 96$ & 4 & & & & & & 262.0 & ND & 46 & 8584 \\
\hline $11 / 21 / 96$ & blank & & & & & $<1$ & ND & ND & $<10$ \\
\hline
\end{tabular}

Remarks:
Total $\mathrm{K} \quad$ Alkalinity to $\mathrm{pH} 4.5$ as $\mathrm{mg} \mathrm{CaCO}_{3} / \mathrm{L}$
Pal K Alkalinity to $\mathrm{pH} 8.3$ as $\mathrm{mg} \mathrm{CaCO}_{3} / \mathrm{L}$
Acid Acidity to $\mathrm{pH} 8.3$ as $\mathrm{mg} \mathrm{CaCO} / \mathrm{L}$

Analytic Method Codes:

* $\quad \mu \mathrm{g} / \mathrm{L}$, Inductively coupled plasma

** $\quad \mathrm{mg} / \mathrm{L}$, Ion chromatography

*** $\quad \mathrm{mg} / \mathrm{L}$, Titrimetric

ND No detection 
SECTION 5

UNDERGROUND PLACEMENT

\author{
Dr. Y.P. CHUGH \\ CO-PRINCIPAL INVESTIGATOR
}

MR. ERIC POWELL
RESEARCHER

MR. GARY WANGLER

RESEARCHER 


\title{
MANAGEMENT OF DRY FLUE GAS DESULFURIZATION BY-PRODUCTS IN UNDERGROUND MINES
}

\author{
Technical Progress Report - October 1, 1996 - December 31, 1996
}

\section{UNDERGROUND PLACEMENT}

\section{Pneumatic Injection}

\section{Design of Equipment.}

Modifications and minor changes were made to the design of the pneumatic injection equipment, especially in the performance monitoring and recording equipment. The selection of pressure transducers and flow meters with $4-20 \mathrm{~mA}$ outputs for recording with a multi channel recorder has been co-ordinated with the mining engineering department laboratory research staff to ensure the recorded results can be transferred to a PC program. We are awaiting the availability of the DOE 486 PC, previously used in the tests at the University of Pittsburgh, prior to making the final selection of pressure, flow and temperature transducers.

As the delivery arrangements of the water supply required for the additional mixing had not been determined, i.e. by tanker truck, pipeline or direct pumping from an adjacent lake, it was decided to incorporate a water tank with the equipment. This was designed with a 30 minute storage capacity and the air reservoir skid extended to support the $8 \mathrm{ft}$. $88 \mathrm{ft}$. $\times 4 \mathrm{ft}$ high steel tank. This arrangement will provide flexibility to accommodate which ever method is used to supply the water to the pneumatic injection equipment.

\section{Purchasing and Delivery of Components.}

The balance of the components not purchased in the previous quarter were procured and delivered to the assembly bay at the Illinois Coal Research Center, Carterville. All items were inspected prior to assembly, especially those involving air pressure such as the receiver vessels, safety relief and blow out valves, air delivery hoses, etc. Support equipment such as the rope slings and spreader bar for unloading at the mine location were also received. Close liaison with the SIUC Purchasing Department was continued to ensure competitive prices were obtained for the numerous vender supplied items, as had been the practice for the procurement of custom fabricated items during the previous quarter. Arrangements were made for the immediate purchase of small items which may have been overlooked in the parts list, or due to last minute modifications, such as bolts, nuts, pipe fittings, air hoses, etc. 


\section{Assembly of Major Components.}

The major components were delivered in early December and work on the assembly commenced immediately. The material conveying tanks were installed on the skid base and the feed screws and end housings fitted into the tanks. The water tank and compressed air receivers assembled on the second skid base. Work commenced on fitting the pipework and butterfly valves. At the end of this period the hydraulic/pneumatic control console was delivered and will be installed at the start of the next quarter together with the hose connections. It is expected that the assembly of the pneumatic injection will be completed by the fourth week in January. Figure 5.1 is an overall view on the pneumatic placement unit being assembled at the Carterville facility. Figure 5.2 is a close-up view of the ribbon screw conveyors in the fly-ash vessels.

\section{Meetings.}

Presentations were made at the USIC Steering Committee meeting, outlining the proposed application of the pneumatic injection equipment and the air blast technique to transport the fill material over the required distance of 300 feet. A presentation was made to the DOE-METC Meeting at the University of Kentucky at Lexington, including slides of the general arrangement of the injection equipment.

Meetings were held at Peabody No. 10 Mine with Graves Brothers to select the site for assembling the mixing plant in relation to the boreholes, both for the hydraulic paste and pneumatic filling techniques.

\section{Research Mine Model of Pneumatic Injection Technique.}

To determine the behavior of the material flow as it transgresses through the mine entries and cross cuts from the borehole and the movement of the material from the effect of the air blast, a scale model has been constructed. This consists of several sections of fabricated steel troughs, 4 " deep $\times 13$ " wide, with a total length of 16 feet and one cross cut of four feet. The sections are bolted together and can be arranged in various configurations. The top cover, or simulated mine roof, consists of glass sheets held in place with screw clamps for easy removal. The mining laboratory air compressor which produces 5 cubic feet of air at 100 psig is being utilized as the air source both for conveying and the blast air.

The material (fly-ash) feed rate is controlled by varying the air flow through the holding tank, which is fitted with a membrane for fluidization. Initial tests have shown an indication of the channeling at the roof level as we would expect in the underground entries. However modifications may be required to the material feeding arrangement for closer control. 


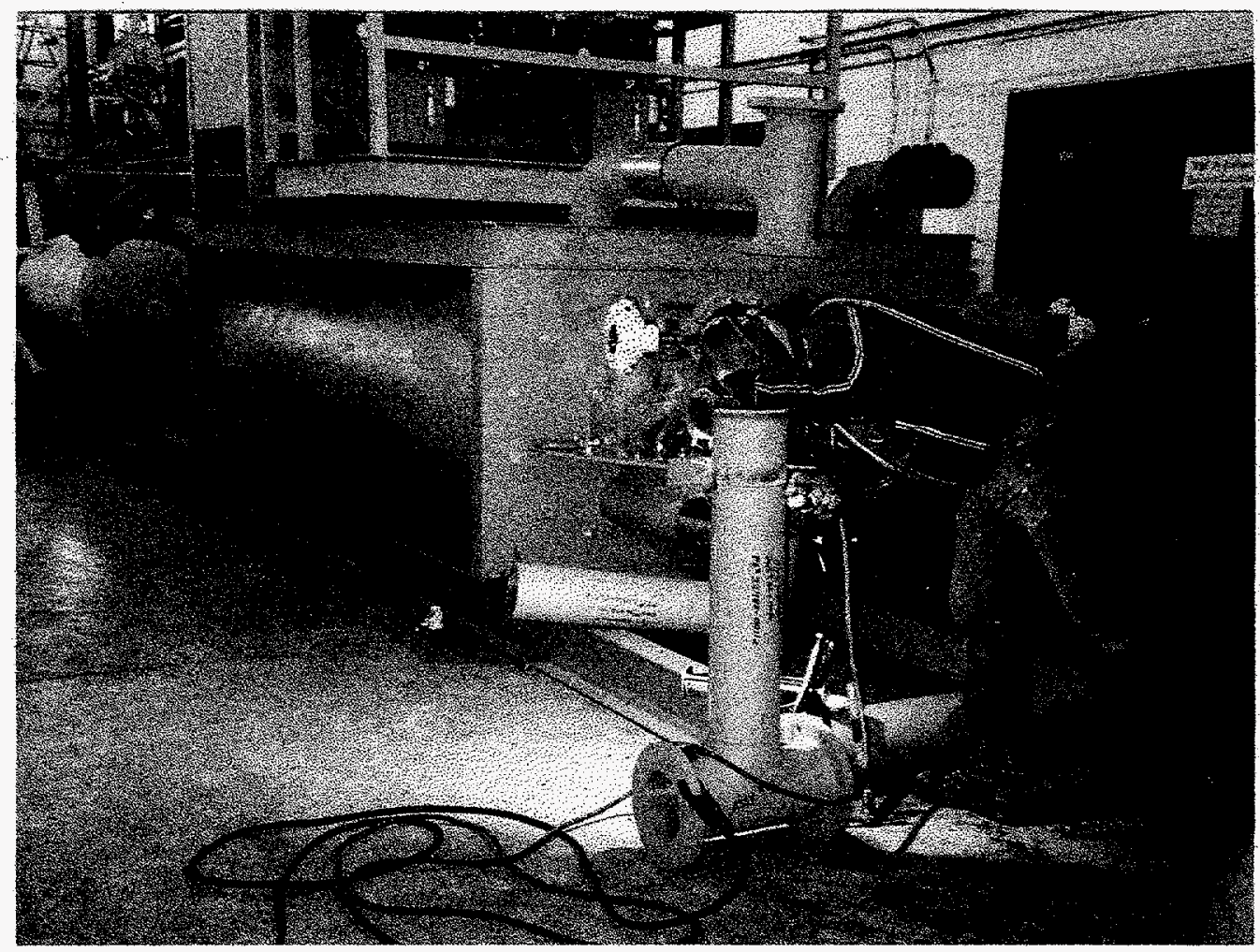

Figure 5.1 Overall view of Pneumatic Placement Unit Under Construction.

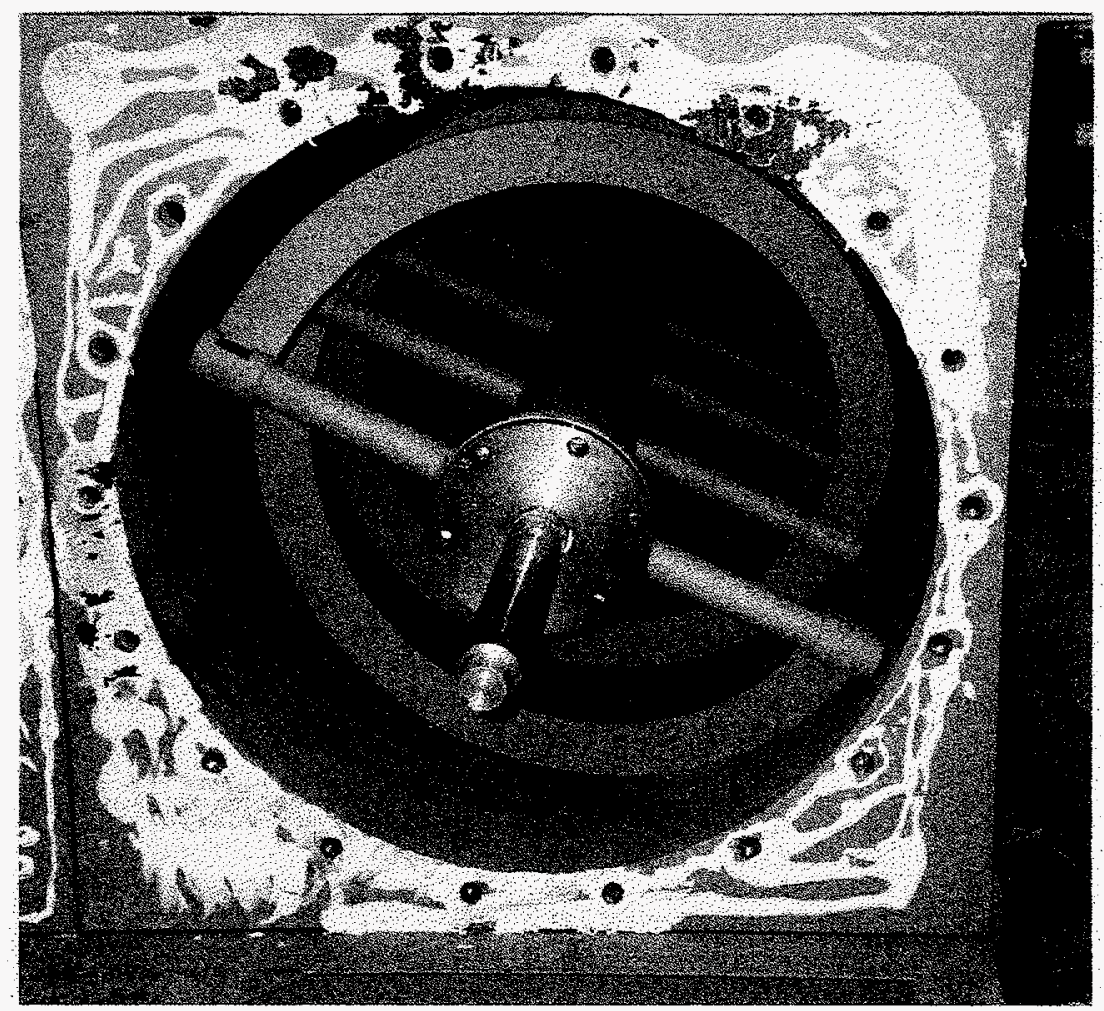

Figure 5.2 Close-Up of the Ribbon Screw Conveyors in the Fly Ash Vessels. 


\section{Hydraulic Injection}

\section{Design of Equipment}

The design of the hydraulic injection equipment did not require the design and drawing that was essential for the pneumatic injection equipment. Rather, the hydraulic equipment can be fabricated from readily available components. The principal requirement is a mixer or pug mill for the thorough mixing of the fly ash, scrubber sludge, and lime waste that composes the hydraulic mixture to be injected underground. Also essential is a high capacity concrete pump, again, a readily available item.

As previously reported, a mixing plant was located which was owned by Montgomery County, Illinois. The plant had been used by the Montgomery County highway department to mix coal combustion by-products to be used for county road repairs. The mixing plant was located at the Central Illinois Power Service (CIPS) Coffeen electrical generating plant, from which Montgomery County obtained the necessary combustion by-products. However, apparently Montgomery County had decided to use other materials for road repair, and wished to dispose of the mixing plant.

After several visits to the mixing plant, and careful inspection by program personnel, the plant was deemed suitable for use in the hydraulic injection phase of the program. Accordingly, the plant was acquired by purchase. As the plant included a pug mill, along with other components for the controlled mixing of combustion by-products, little other design of the hydraulic system was necessary. In short, the acquisition of the mixing plant resulted in an already-designed and fabricated system suitable for the hydraulic injection phase of the program.

\section{Delivery of the Plant}

Arrangements were made for Peabody Coal Company, under their sub-contract with SIUC, to dismantle the mixing plant at its original location at the CIPS Coffeen plant, transport it to the demonstration site (Peabody No. 10 mine) and re-assemble it with minor modifications. This was undertaken during the later part of the quarter, and by the end of the quarter all segments of the plant were at the demonstration site. However, the Christmas holidays and adverse weather conditions prevented the completion of the plant re-assembly. It is anticipated that the mixing plant will be totally re-assembled by the end of January, 1997.

Other components needed for the hydraulic injection will be acquired through rental at the time of the shake-down demonstration. The most critical item is the highcapacity concrete pump. The pump has been rented for use on other SIUC research projects, and thus program personnel are familiar with the pump and its operation. There is no need to rent the pump until such time as it is needed for the shake-down test. 


\section{SECTION 6}

\section{PLANS FOR NEXT QUARTER}

Mr. Edwin M. Thomasson

PROGRAM MANAGER 


\title{
MANAGEMENT OF DRY FLUE GAS DESULFURIZATION BY-PRODUCTS IN UNDERGROUND MINES
}

\author{
Technical Progress Report - October 1, 1996 - December 31, 1996
}

\section{PLANS FOR NEXT QUARTER}

The principal objective for the next quarter (January 1 - March 31, 1997) is to conduct "shake-down" tests of both the pneumatic and hydraulic injection equipment. This will entail transporting the pneumatic injection equipment from the SIUC Carterville facility to the demonstration site at the Peabody No. 10 mine, and the drilling of suitable boreholes for the shake-down tests. The tests will involve the underground placement of approximately 800 tons of coal combustion by-product mixtures by each technology (i.e., by both pneumatic and hydraulic technologies).

Also during the next quarter an application for the continuation of the program into Phase III will be developed and submitted to appropriate Department of Energy personnel. A Test Plan for Phase III will also be developed and submitted to DOE.

A more concentrated effort to modify the standard steel intermodal container to make it suitable for the handling and transporting of coal combustion by-products will be undertaken. This part of the program has been delayed due to some unanticipated circumstances, but it is believed that the project can now go forward with the cooperation of the Illinois Central Railroad.

Topical reports on (1) Residues Characterization and Mix Development, and (2) Identification/Assessment of Technology for Transport/Handling of FGD Residues will be completed during the quarter and submitted to the Department of Energy.

Also, Dr. Hasan Sevim will deliver a paper entitled "Promoting Illinois Coal Utilization Through Underground Disposal of Combustion Products" at the Annual Meeting of the Society of Mining Engineers in Denver, Colorado on February 23-27, 1997. 


\title{
PROMOTING ILLINOIS COAL UTILIZATION THROUGH UNDERGROUND DISPOSAL OF COMBUSTION BY-PRODUCTS
}

\author{
by \\ Hasan Sevim \\ Department of Mining Engineering \\ Southern Illinois University at Carbondale \\ Carbondale, IL 62901 \\ Phone: 618-453-7922 \\ Fax: 618-453-7455 \\ Email: sevim@engr.siu.edu
}

\begin{abstract}
The utilization of high sulfur Illinois coal by utility plants has been decreasing since the enactment of 1990 Clean Coal Air Act Amendment. Illinois coal producers can make their coal saleable by offering the choice of taking the combustion by-products from the utility plants and placing them in the abandoned sections of their mines. To be competitive, however, coal producers need to select an environmentally acceptable transportation-handling-placement system through which the cost-per-ton of by-product disposed can be minimized. In this paper, a few such systems are presented. The favorable operating range of each system is determined in terms of distance and tonnage. A case study is given whereby cost analysis is conducted for an underground coal mine receiving by-products from two Illinois utility plants.
\end{abstract}

\section{INTRODUCTION}

In order to comply with Phase I of the 1990 Clean Air Act Amendment (CAAA) a number of coalfired power generating plants have switched to low-sulfur western coal. The most recent statistics indicate that the utility purchases of Illinois coal dropped from nearly 54 million tons in 1990 to 41 million tons at the beginning of the implementation of Phase I in 1995. It is projected that this amount will drop to 37.5 million tons by the year 2000 when Phase II begins (Keefe, 1996). Demand for utility coal is expected to increase after 2000 in response to projected growth in coal-fired power generation in central and eastern regions of the United States (Philo et al., 1995). To prevent further losses and capture a respectable share of the future utility coal market, Illinois coal producers should develop new strategies and policies.

One of the strategies could be the offering of the underground placement of combustion by-products $(\mathrm{CCB})$ into the old workings of the underground coal mines as an incentive in the coal contracts. The placement can be done from the surface through boreholes into the sealed sections of an active mine or into an abandoned mine. This alternative of by-product placement can benefit both the utility plant and the mine. Currently, approximately 90 million tons of CCB (fly ash, bottom ash, boiler slag, scrubber sludge) are generated annually by the U.S. utility industry. Only 20 million tons finds utilization, the rest is disposed of in surface facilities, or landfills, near power plants (ACAA, 1993). Surface disposal is becoming more and more expensive, especially in and around urban areas. Underground disposal of CCBs by the coal supplier can be a long-waited solution for the utility plants. 
For the coal supplier, the major benefit of underground placement is the increase in coal sales. There could also be additional benefits in reducing subsidence and mine drainage -- two major problems resulting from underground coal mining -- by backfilling the voids with the CCBs (Meiers et al., 1995).

Recognizing these potential advantages, the U.S. Department of Energy (DOE) entered into a cooperative research program with the Southern Illinois University (SIU) to investigate the engineering, environmental, and economic feasibility of disposing by-products into abandoned sections of underground coal mines. The project is progressing in six branches and is expected to be completed in 1997 (Chugh et al., 1996). The six branches are: 1) By-Products and Mix Characterization, 2) Materials Handling and System Economics, 3) Underground Placement, 4) Environmental Monitoring and Assessment, 5) Health and Safety Issues and Permitting, and 6) Field Demonstration.

During the conduct of the Materials Handling and Economics study in the above research program, it was set forth that, for underground placement to be economically competitive, the transportation/handling/placement costs had to be minimized. An engineering design and a cost model were developed for this purpose and different modules were keyed into an interactive software package (Sevim et al., 1995).

The Engineering design model was developed for the determination of number of transport units, silo capacity, loading and unloading rates, placement system capacity, number of shifts, etc., for a given case defined by a distance-tonnage combination. The cost model is for the determination of the operating and capital costs and cost-per-ton of by-product transported and placed. This model is based on the "After-Tax Cost" computation method which takes into account depreciation and taxation.

\section{CCB TRANSPORTATION AND HANDLING TECHNOLOGIES}

Several transportation and handling technologies were first evaluated in order to find those that are environmentally acceptable. These technologies can be classified under two categories:

1. Existing Technologies:

1.1 Pneumatic Trucks (PT)

1.2 Pressure differential rail cars (PD-car)

1.3 Open Hopper Coal Cars

1.4 Tarped Rear-Dump Trucks

1.5 Bottom-Dump Container Trucks

2. Adaptable and Futuristic Technologies:

2.1 Collapsible Intermodal Containers (CIC)

2.2 Cylindrical Intermodal Tanks (CIT)

2.3 Intermodal Steel Containers

2.4 Coal Hopper Cars with Automatic Retractable

Tarping

2.5 Covered Hopper Cars - Grain Cars

The first two technologies in each category were found to be environmentally friendly, therefore, operating policies for these technologies were developed and adapted into the interactive software (Chugh et al., 1996). 
The pneumatic trucks (PT), also referred to as bulk tank trucks, are widely used in transporting low density dry flowable powder and granular materials as well as high density materials such as cement, limestone and fly ash. In the developed fly ash transportation scenario, the trucks will be loaded from the fly ash bin of the plant and they will deliver the material directly to the placement point at the mine site. There, the pressure necessary for offloading the fly ash into the injection hopper will be supplied either by a blower mounted on the truck or by a stationary blower. These trucks are approximately 20-25 tons in capacity and can offload in about 20-25 minutes (Freitag et al., 1991).

The Pressure Differential Rail Car (PD-car) is a special type of rail car used to handle powdered materials. They are operated under the principle of pressure differences between the car and the container to which the product is discharged. When a PD-car is pressurized to approximately 5 psi or more, the outlet valves are opened to form a steady flow of material into the truck until all the material in the compartment is cleared. In the developed transportation scenario, one set of cars will be filled at the power plant while the other set will be emptied at the mine. When all the cars at the plant are filled, either a "local train" or a "unit coal train" will take them to the mine. The empty PD cars will be delivered to the plant in the same way. At the mine, the product in the PD cars will be transferred into a silo with the aid of a stationary blower. Delivery from the silo to the injection site can be done either by pneumatic trucks, or by regular dump trucks if the silo is equipped with a pugmill to wet the residue to prevent fugitive dust.

The Collapsible Intermodal Containers $\left(\mathrm{CIC}^{\mathrm{TM}}\right)$ are made of rubber coated aramid and nylon fabric with polyester webbing. They are patented by SEEC Inc., one of the partners in the DOE-SIU research program. The CICs are collapsible storage bins that are portable and intermodal -- designed to ride inside coal cars, barges and trucks. Those CICs made to transport fly ash by riding in coal cars have a height of 120 inches, diameter of 110 inches and a 19-inch filling port. For ash of $60 \mathrm{lb} / \mathrm{ft}^{3}$ bulk density, the CIC capacity is about 20 tons.

In the CIC scenario, there will be coal transportation from mine to plant and CCB transportation from plant to mine. This mode of operation will provide "backhaul" charge for CCB transportation which may be substantially less than the "fronthaul" charge. In this scenario, a coal train will arrive at the plant and offload coal into an under-track bin. Next, the CICs which have already been filled with fly ash and staged along the rail will be lifted, one at a time, by an overhead crane and placed into the bays of the empty coal cars. Four CICs will occupy a car, each taking one of the four bays of a typical coal car. When all the CICs are loaded, the train will leave for the mine.

At the mine site, an overhead crane will lift the CICs, one at a time, and will place them on the concrete pad along the rail track.. These CICs will then be loaded on tote trailer(s) by the same crane and transported to the injection site. There, the ash will be offloaded into the hopper of the injection system by the use of a vacuum system designed for the CICs. After collecting 25-40 empty CICs, they will be transported back to the plant in a trailer.

At the plant, the empty CICs will be retrieved from the trailer with the help of a small forklift and placed, one at a time, on a specially designed trailer and pulled under the fly ash silo by a tractor. There, it will be filled by gravity similar to filling a pneumatic truck. It will then be transported back to the rail site where the trailer will pull under the overhead crane so that the CIC can be lifted and staged along the track, and kept there until the coal train comes back from the mine to pick them up.

The Cylindrical Intermodal Tanks (CIT) are made of either steel or aluminum and have a volume of approximately 6400 gallons. They are currently being used in transporting liquids and liquefied gases. The capacity of a tank will be approximately 20 to 25 tons assuming an average density of $60 \mathrm{lbs} / \mathrm{ft}^{3}$ for coal combustion residue. Since they are cylindrical, the bridging and sticking problems that occur in rectangular containers when handling powdered material like fly ash, or damp material like scrubber sludge, can be eliminated. These tanks may be mounted in steel frames to facilitate handling. 
In the CIT transportation scenario, an empty tank will be placed on a trailer with the aid of a piggy packer (a specialized crane) and shuttled to residue silo where it will be filled like a pneumatic truck. At the rail siding, the piggy packer will lift the filled tank and stage it along the railroad on a concrete pad. When the train arrives, the piggy packer will lift these tanks again one by one and place them on the flat bed rail cars. The length of these cars are suitable to handle 3 of these tanks on one car. When all the CITs are loaded, the train will leave for the mine.

At the mine site, the tanks will be lifted again by a piggy packer and placed on the concrete pad. After the unloading is completed, the same packer will lift the tanks one by one and place them on a trailer to be taken to the injection site. The unloading of the by-product into the injection hopper will be done by elevating the head of the tank with the aid of a hydraulic jack mounted on the trailer. The gate of the tank will then be opened and the content transferred into the injection system hopper.

The empty tanks will be staged at the rail siding and will be waiting for the train to pick them up. After delivering the empty tanks to the plant another cycle will restart.

\section{FAVORABLE OPERATING RANGES OF TECHNOLOGIES}

The selection of one technology over the other is based on the system cost which is a function of the transportation distance and tonnage. Therefore, we have to establish economically favorable operation ranges for each technology in terms of distance and tonnage.

In southern and central Illinois, annual CCB production range between 50,000 to 200,000 tons, and typically plants are 50 to $320 \mathrm{~km}$ ( 30 to $200 \mathrm{miles}$ ) away from underground mines. Therefore, all evaluations were conducted for distance-tonnage combinations within these ranges. Using the developed software, cost-per-ton figures were calculated for cases of 50,000 tons, 100,000 tons, and 200,000 tons annual by-product. The distances of transportation considered for each annual tonnage were 50, 160, and $320 \mathrm{~km}(30,100$, and 200 miles), respectively. Therefore, nine runs were conducted for each of the four selected transportation and handling technologies, namely; PT, PD-car, CIC and CIT.

The outcomes of the cost analyses are summarized in Figures 1, 2, and 3. The cost-per-ton values shown in these figures reflect the costs of transportation, handling, and underground placement of the byproducts.

At 50,000 tons (Fig. 1), the PT technology gave lower costs than the other technologies up to a distance of approximately $200 \mathrm{~km}$ ( 125 miles). The PD-car and the CIT technologies indicated almost the same cost for all distances, and they were better than the CIC technology up to a distance of approximately $225 \mathrm{~km}$ (140 miles). After $240 \mathrm{~km}$ ( $150 \mathrm{miles})$, there were no significant differences among the PD-car, CIT, and CIC technologies and they were all lower than the cost provided by the PT technology. The steeper slope of the PT cost line when compared to others is due to increased cycle time at longer distances, which imposes the addition of new truck units in the fleet in order to handle the same 50,000 tons per year by-product.

At 100,000 tons (Fig. 2), the PT technology gave lower costs then the others up to a distance of 160 $\mathrm{km}$ (100 miles). The PD-car, CIT, and CIC technologies indicated almost the same cost up to a distance of $160 \mathrm{~km}$ (100 miles), after which the CIC technology gave lower costs.

At 200,000 tons, the picture was almost identical to that of 100,000 tons, except that the PT technology was better than the others up to only $110 \mathrm{~km}$ ( 70 miles). This is due to the fact that the economies of scale favors the PD-car, CIT, and CIC technologies over the PT technology. In other words, as the production rate increases the cost given by the PT technology remains almost unaffected whereas the costs by the other three technologies decreases significantly. Therefore, it can be concluded that the PT technology is sensitive to distance but insensitive to tonnage, whereas the opposite is true for the other three technologies. 
The findings in "favorable range" study can now be used in a case study.

\section{CASE STUDY}

Peabody No. 10 mine in Pawnee, Illinois, can be considered a representative case for potential disposal sites in Illinois. Pneumatic and hydraulic placement systems developed in the cooperative research program will be demonstrated at this site in the Spring of 1997.

This mine, located approximately $32 \mathrm{~km}$ (20 miles) south of Springfield, Illinois, was opened in the 1950s and operated until August, 1994. Room-and-pillar mining method had been employed utilizing continuous miners to mine Herrin (No. 6) coal seam. The mine is about $106 \mathrm{~m}$ (350 ft) deep with a seam thickness varying from $1.83 \mathrm{~m}$ to $2.44 \mathrm{~m}(6$ to $8 \mathrm{ft})$.

For best results in pneumatic placement, the researchers in "Residues and Mix Characterization" group of DOE-SIU research program have found that the mix should be composed of $80 \%$ FBC fly ash and $20 \%$ spent-bed ash by weight. Similarly, the optimum mix for hydraulic placement should be composed of $55 \%$ scrubber sludge, $40 \%$ fly ash, and $5 \%$ lime waste (Chugh et al., 1996).

The source of FBC fly ash and spent-bed ash for the pneumatic placement will be the Archer Daniel Midland Company (ADM) power generating plant at Decatur, Illinois. The source of scrubber sludge and fly ash for the hydraulic placement will be the Dallman plant of City, Water, Light and Power (CWLP) Company in Springfield, Illinois. The locations of these two plants and the Peabody No. 10 mine are shown in Figure 4. As seen, the mine is about $40 \mathrm{~km}$ (25 miles) south of Dallman Plant and $104 \mathrm{~km}$ (65 miles) southwest of ADM Plant.

The case study was conducted for both the pneumatic and hydraulic placement. Consistent with the findings in "Favorable Range" study discussed above, only truck transportation was considered since the distances of both plants to the mine favored truck transportation over the others. It is noted that scrubber sludge which contains about 15 to $20 \%$ water cannot be transported in pneumatic trucks. Therefore, reardump trucks were used for this product.

A base case was defined at 100,000 tons production rate. However, to reveal the effect of economies of scale, two additional cases were considered around the base case; one for 50,000 tons and the other for 150,000 tons. Furthermore, to isolate the transportation cost from that of the placement, the entire system was re-evaluated for all production rates by excluding the placement unit.

The following additional assumptions were made:

$\begin{array}{lrl}\text { Injection through one borehole } & : 10,000 \text { tons } \\ \text { Project life } & : 10 \text { years } \\ \text { Effective tax rate } & : 40 \% & \\ \text { Discount rate } & : 12 \%\end{array}$

First, the Dallman case (hydraulic placement scenario) was evaluated. The total capital investment, including the hydraulic placement unit, was estimated to be approximately $\$ 1$ million whereas the annual operating cost $\$ 1.142$ million. The cost-per-ton figures are shown in Figure 5. The lower cost line corresponds to scenarios excluding the hydraulic placement unit, hence, the placement cost at any production rate can be read as the vertical difference between the two lines. For instance, at 100,000 tons, the transportation and handling cost with and without placement are $\$ 5.98$ and $\$ 3.29$ per ton, respectively. The placement cost, therefore, is $\$ 2.69$ per ton $(5.98-3.29)$. 
The cost-per-ton figures obtained for The ADM case (pneumatic placement scenario) are shown in Figure 6. At 100,000 tons, the transportation and handling cost with and without placement are $\$ 8.13$ and $\$ 5.77$ per ton, respectively. The pneumatic placement cost, therefore, is $\$ 2.36$ per ton ( $\$ 8.13$ \$5.77). This is slightly lower than the cost of hydraulic placement due to lower capital outlay of pneumatic placement unit. For a capacity of $60 \mathrm{tph}$, it was estimated that the pneumatic and hydraulic units would cost $\$ 133,000$ and $\$ 250,000$, respectively.

The slight upward trend when production increases from 100,000 tons to 150,000 tons is due to the increase in the truck fleet size from 8 trucks to 13 trucks to cover the 65-mile distance. In Dallman case, however, the fleet size increased from 5 trucks to 6 trucks, because the transportation distance was only 40 $\mathrm{km}$ (25 miles).

\section{CONCLUSIONS}

Illinois coal producers have been loosing ground to western low-sulfur coal in the utility market. They have to find new ways of marketing the coal before the demand starts swinging up after the year 2000 as projected. In this paper, the offering of underground placement of CCBs in the old workings of underground coal mines is presented as a potential incentive to win coal contracts from utility plants. The cost of such placement will depend on the transportation/handling/placement technology as well as the distance of transportation and production rate. The engineering design and cost model developed in the DOE-SIU collaborative research program can help coal producers (or utility plants, or contractors) in selecting the appropriate technology and in minimizing the costs involved.

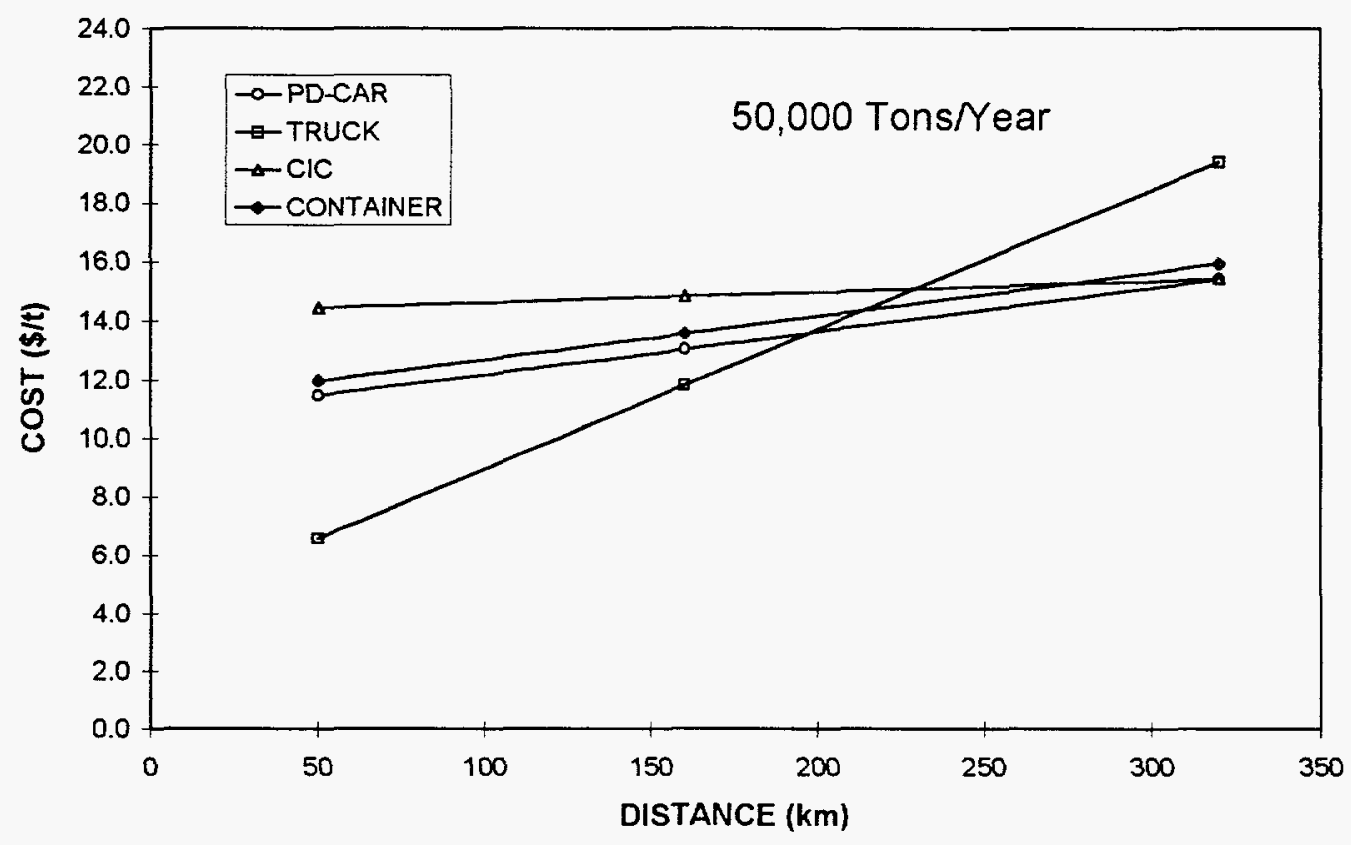

Figure 1. Cost-per-ton in four different transportation technology when 50,000 tons of by-product are transported and placed 


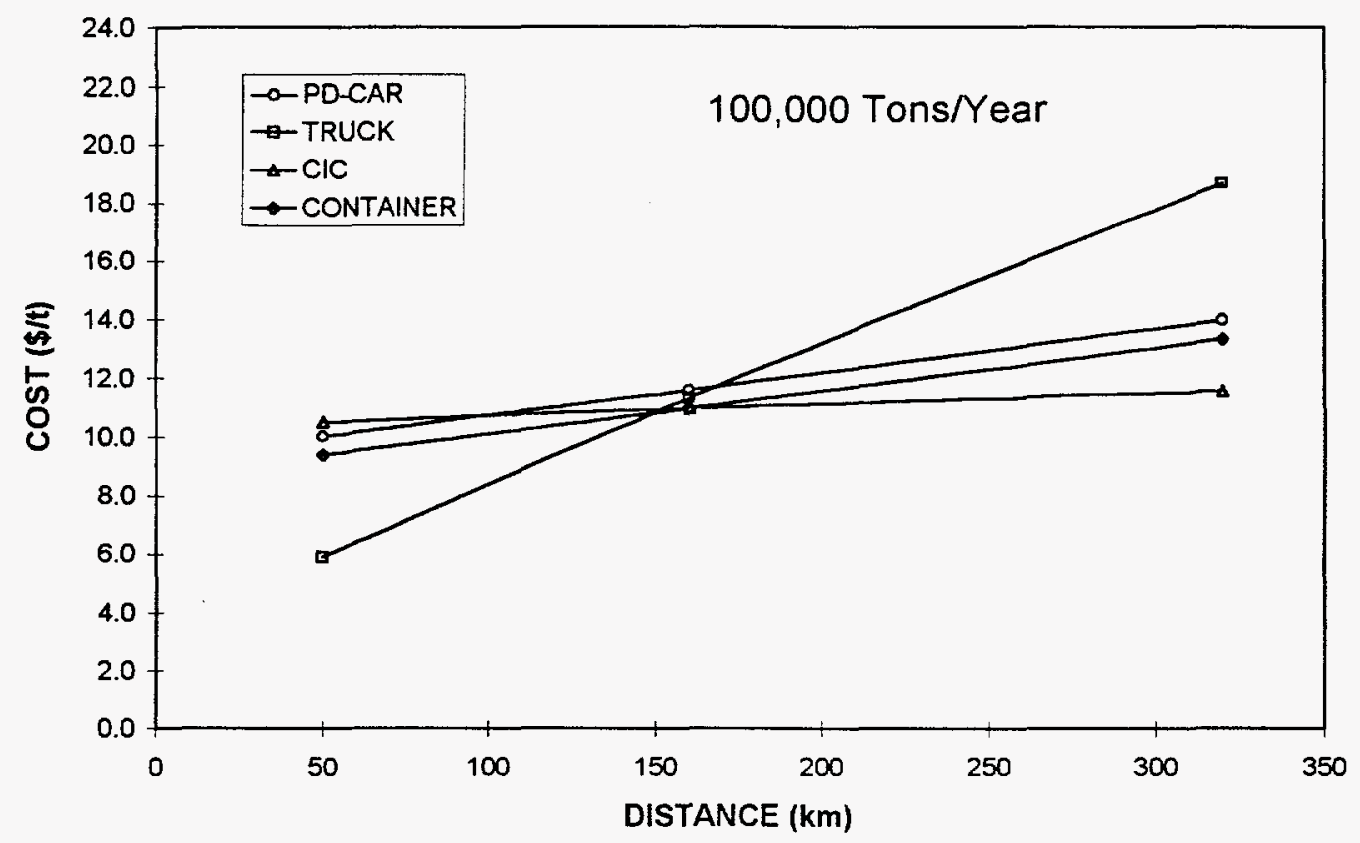

Figure 2. Cost-per-ton in four different transportation technology when 100,000 tons of by-product are transported and placed

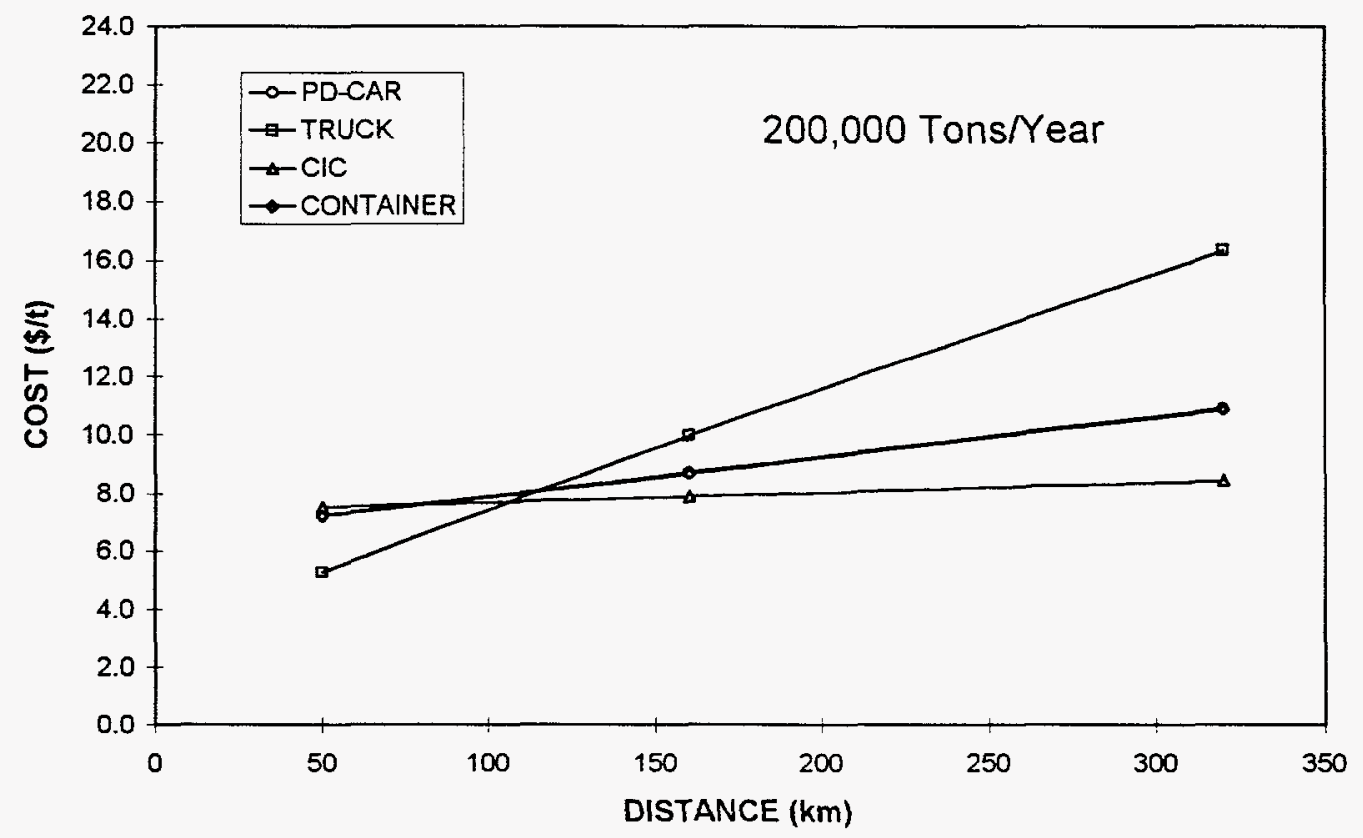

Figure 3. Cost-per-ton in four different transportation technology when 200,000 tons of by-product are transported and placed 


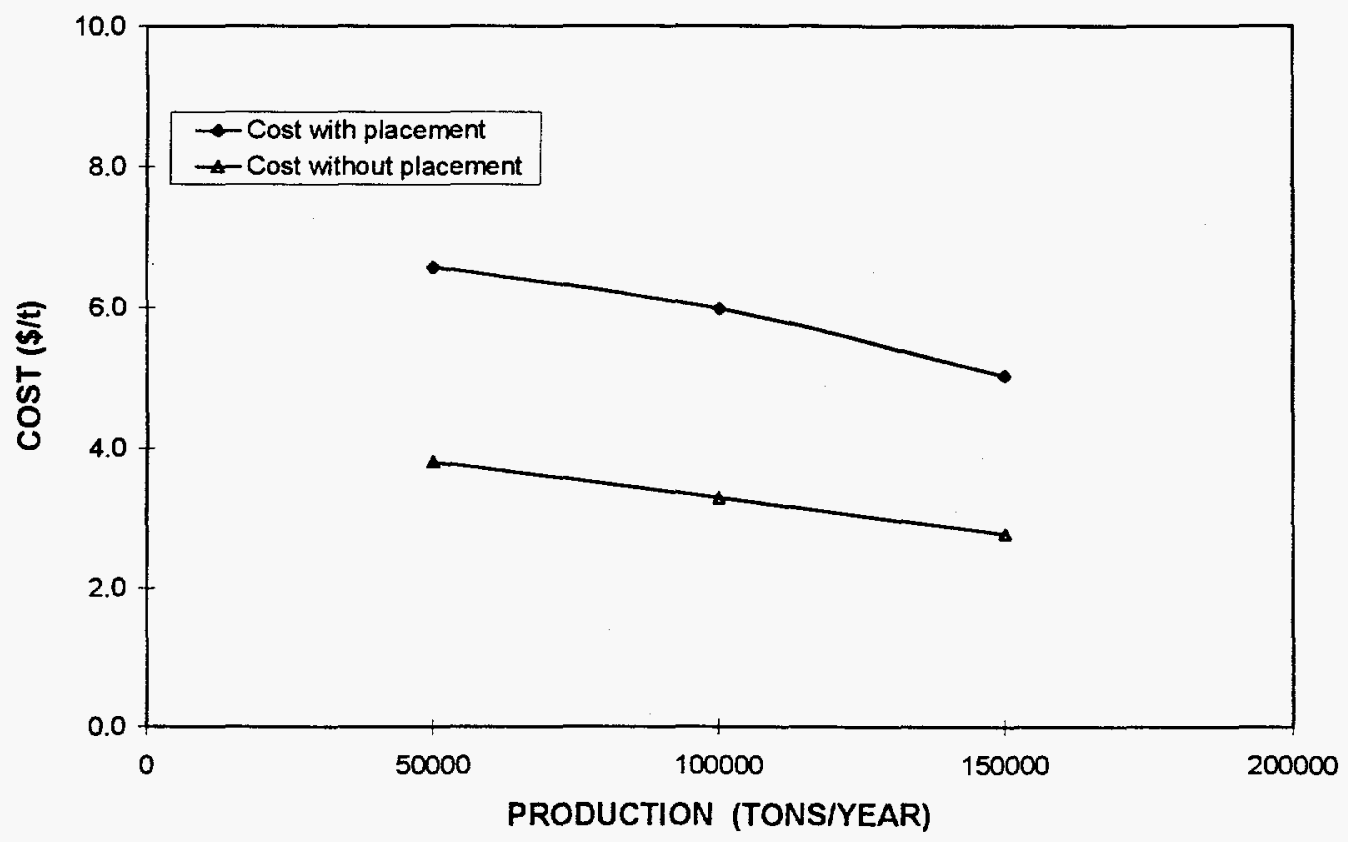

Figure 5. Cost-per-ton for by-products transported from Dallman plant to Peabody No.

10 mine

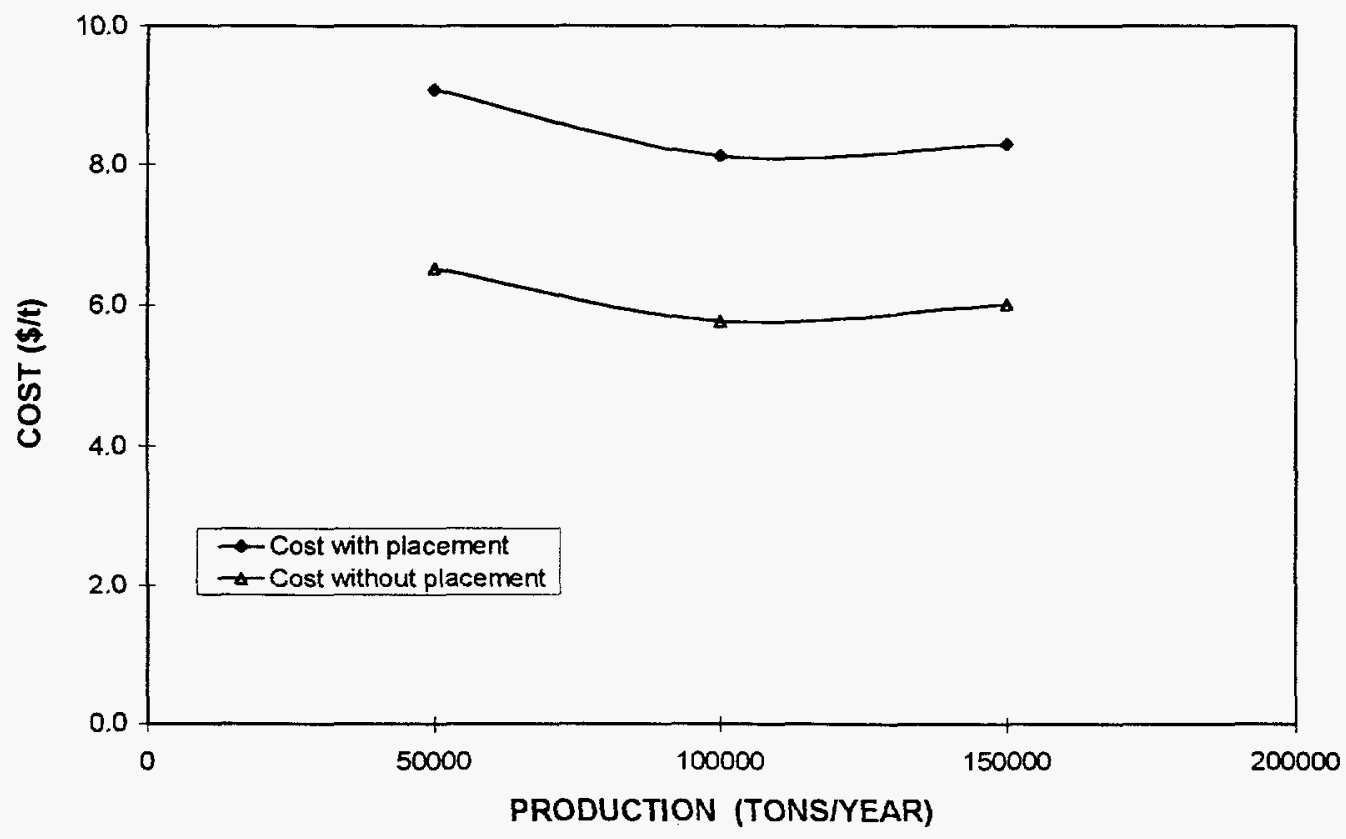

Figure 6. Cost-per-ton for by-products transported from ADM plant to Peabody No. 10 mine 


\section{ACKNOWLEDGMENT}

The work reported in this paper is a part of a research project entitled " Management of Dry Flue Gas Desulfurization By-Products in Underground Coal Mines" funded by the U.S.D.O.E. under grant no. DEFC21-93MC 30252. Their support and cooperation is gratefully acknowledged.

\section{REFERENCES}

American Coal Ash Association (ACAA) (1993) Coal Combustion By-products (CCB) - Production and Use. A single page flier from ACAA, Alexandria, VA.

Chugh, P., S. Esling, B. Paul, H. Sevim, E. Thomasson, X. Yuan, 1996, "Management of Dry Flue Gas Desulfurization By-Products in Underground Mines", Annual Technical Progress Report, DE-FC2193MC30252, U.S. Department of Energy, Morgantown Research Center, WV.

Keefe E. D., personal communication, May 8th, 1996, Illinois Department of Commerce and Community Affairs, Springfield, Illinois.

Meiers, J., D. Golden, R. Gray, and W. Yu, 1995, "Fluid Placement of fixated scrubber sludge to reduce surface subsidence and to abate acid mine drainage in abandoned underground coal mines", Proceedings of the 1995 International Ash Utilization Symposium, October 23-25, Lexington, Kentucky, Section VI.

Freitag, A. J., T. E. Hemminger, and G. Garrison, 1991, "Coal Combustion Ash Disposal Underground Injection of Fly Ash into Mined-Out Portions of Coal Mine", Paper No. 91-37.9, 8th Annual Meeting of Air \& Waste Management Ass., Vancouver, British Columbia, June 16-21.

Philo R. G., D. E. Keefe, D. W. South, and K. A. Bailey, 1995, Outlook for the Illinois Coal Industry, Illinois Department of Commerce and Community Affairs, Springfield, Illinois.

Sevim H., D. Lei, and S. Gwamaka, 1995, "An Interactive Software for the Evaluation of Residue Transportation and Handling Alternatives", In Proceedings of 1995 International Ash Utilization Symposium, Lexington, Kentucky, October 23-25, Section VIII. 